MATHEMATICS OF COMPUTATION

Volume 75, Number 255, July 2006, Pages 1043-1065

S 0025-5718(06)01833-3

Article electronically published on May 1, 2006

\title{
ANALYSIS OF SOME LOW ORDER QUADRILATERAL REISSNER-MINDLIN PLATE ELEMENTS
}

\author{
PINGBING MING AND ZHONG-CI SHI
}

\begin{abstract}
Four quadrilateral elements for the Reissner-Mindlin plate model are considered. The elements are the stabilized MITC4 element of Lyly, Stenberg and Vihinen (1933), the MIN4 element of Tessler and Hughes (1983), the Q4BL element of Zienkiewicz et al. (1993) and the FMIN4 element of Kikuchi and Ishii (1999). For all elements except the Q4BL element, a unifying variational formulation is introduced, and optimal $\mathrm{H}^{1}$ and $\mathrm{L}^{2}$ error bounds uniform in the plate thickness are proven. Moreover, we propose a modified Q4BL element and show that it admits the optimal $\mathrm{H}^{1}$ and $\mathrm{L}^{2}$ error bounds uniform in the plate thickness. In particular, we study the convergence behavior of all elements regarding the mesh distortion.
\end{abstract}

\section{INTRODUCTION}

Considerable attention has been paid to the design and analysis of low order locking-free elements for the Reissner-Mindlin plate model over the past two decades, see, e.g., 10, 13, 14, 20. However, there is still a gap between plate elements commonly used in engineering and those for which a sound mathematical theory exists. Even worse, the existing analyses are mostly confined to either triangular elements or rectangular elements, but exclude most widely used quadrilateral elements, like the stabilized MITC4 element of Lyly, Stenberg and Vihinen [27, the aniso-parametrically interpolated MIN4 element of Tessler and Hughes [35, the linked interpolated Q4BL element of Zienkiewicz et al. [39, as well as an element proposed recently by Kikuchi and Ishii 21] (FMIN4). These elements are constructed in different settings and perform extremely well in the benchmark computation, however, a rigorous convergence analysis for them seems lacking except the stabilized MITC4 element [25, 26, 30, and the connections between them are unclear. The connections are known for the corresponding triangular elements [24].

The goal of this paper is to analyze the aforementioned quadrilateral elements and to see which one is already locking-free, and on the other hand which elements with guaranteed stability are close and can be used with minimal modifications. Our results indicate that all these elements are almost the same, or all of them are mutatis mutandis. The MIN4 element is the same as the FMIN4 element if the quadrilateral reduces to a parallelogram. Based on an identity which bridges the kinematically linked interpolation operator [7, 24, 35, 37, 38, 39] and the lowestorder Raviart-Thomas-type interpolation operator [2, 36] (see Theorems 4.1 and

Received by the editor October 6, 2002, and in revised form, February 5, 2005.

2000 Mathematics Subject Classification. Primary 65N30, 74K20.

Key words and phrases. Reissner-Mindlin plate, stabilized methods, locking-free.

(C)2006 American Mathematical Society

Reverts to public domain 28 years from publication 
4.2), we introduce a general finite element formulation covering all elements except the Q4BL element, which is known to be a locking element [7, 39]. Assuming that the distance between the mid-points of two diagonals of each quadrilateral $K$ is $\mathcal{O}\left(h_{K}^{1+\alpha}\right)(\alpha \geq 0)$, where $h_{K}$ is the diameter of $K$, we derive the $\mathrm{H}^{1}$ and $\mathrm{L}^{2}$ error bounds for these three elements. We prove that the FMIN4 element converges with optimal rate over the shape regular mesh. The stabilized MITC4 element admits the optimal $\mathrm{H}^{1}$ error over the shape regular mesh, while it attains the optimal $\mathrm{L}^{2}$ error provided that either the plate thickness is commensurable to the mesh size or the mesh satisfies the Bi-Section Condition $(\alpha=1)$. The MIN4 element admits optimal $\mathrm{H}^{1}$ and $\mathrm{L}^{2}$ error bounds for the mildly distorted mesh, i.e., $\alpha=1 / 2$.

On the other hand, we stabilize the Q4BL element by a modification of Auricchio and Lovadina's augmented formulation [7], and derive optimal $\mathrm{H}^{1}$ and $\mathrm{L}^{2}$ errors for the mildly distorted mesh as that in the MIN4 element.

We only consider these four elements for the sake of exposition, while it is believed that our methodology can be applied equally to analyze other quadrilateral Reissner-Mindlin plate elements that have appeared in the literature [8, 9, 38].

The remainder of the paper is organized as follows. The Reissner-Mindlin plate model is described in the next section. We introduce all elements in $\S 3$. The connections among them are established in $\S 4$. The error estimate for all elements except the Q4BL element is carried out in $\S 5$. In $\S 6$, a variant of the Q4BL element is proposed and is proven to be locking-free. Conclusions are drawn in the last section.

Throughout this paper, the generic constant $C$ is assumed to be independent of the plate thickness $t$ and the mesh size $h$.

\section{Reissner-Mindlin Plate MOdel}

Let $\Omega$ be a convex polygon representing the mid-surface of the plate. We assume that the plate is clamped along the boundary of $\Omega$. The bending behavior of the Reissner-Mindlin plate is described by the rotations $\phi$ and the deflection $\omega$, which are determined by the following variational problem: find $(\boldsymbol{\phi}, \omega) \in \boldsymbol{H}_{0}^{1}(\Omega) \times H_{0}^{1}(\Omega)$ such that

$$
\mathcal{B}(\boldsymbol{\phi}, \omega ; \boldsymbol{\psi}, v)=(g, v) \quad \forall(\boldsymbol{\psi}, v) \in \boldsymbol{H}_{0}^{1}(\Omega) \times H_{0}^{1}(\Omega) .
$$

For any $(\boldsymbol{\eta}, w)$ and $(\boldsymbol{\psi}, v)$ in $\boldsymbol{H}_{0}^{1}(\Omega) \times H_{0}^{1}(\Omega)$, the bilinear form $\mathcal{B}$ is defined as

$$
\mathcal{B}(\boldsymbol{\eta}, w ; \boldsymbol{\psi}, v):=a(\boldsymbol{\eta}, \boldsymbol{\psi})+\lambda t^{-2}(\nabla w-\boldsymbol{\eta}, \nabla v-\boldsymbol{\psi}),
$$

with $a(\boldsymbol{\eta}, \boldsymbol{\psi}):=(\mathcal{C E}(\boldsymbol{\eta}), \mathcal{E}(\boldsymbol{\psi}))$. Here $\mathcal{E}(\boldsymbol{\eta})$ denotes the symmetric part of the gradient of $\boldsymbol{\eta}, g$ the scaled transverse loading function, $t$ the plate thickness and $\lambda=E \kappa /[2(1+\nu)]$ with $E$ Young's modulus, $\nu$ the Poisson ratio, and $\kappa$ the shear correction factor. For all $2 \times 2$ symmetric matrix $\boldsymbol{\tau}, \mathcal{C} \boldsymbol{\tau}$ is defined as

$$
\mathcal{C} \boldsymbol{\tau}:=\frac{E}{12\left(1-\nu^{2}\right)}[(1-\nu) \boldsymbol{\tau}+\nu \operatorname{tr}(\boldsymbol{\tau}) \boldsymbol{I}],
$$

where $\boldsymbol{I}$ is the $2 \times 2$ identity matrix. Denote $H_{0}^{1}(\Omega)$ as the standard Sobolev space, and $\boldsymbol{H}_{0}^{1}(\Omega)$ the corresponding space of 2-vector-valued functions. This rule is applicable to other spaces. Define $H^{-1}(\Omega)$ and $\boldsymbol{H}^{-1}(\Omega)$ as the dual space of $H_{0}^{1}(\Omega)$ and $\boldsymbol{H}_{0}^{1}(\Omega)$, respectively. Define $\boldsymbol{V}:=\boldsymbol{H}_{0}^{1}(\Omega), W:=H_{0}^{1}(\Omega)$ and $\boldsymbol{M}:=\boldsymbol{L}^{2}(\Omega)$.

Given the rotations $\phi$ and the deflection $\omega$, the shear stress $\gamma$ is defined as

$$
\gamma:=\lambda t^{-2}(\nabla \omega-\phi) \text {. }
$$


A proper space for the shear stress $\gamma$ is $\boldsymbol{H}^{-1}(\operatorname{div}, \Omega)$, which is defined as the dual space of

$$
\boldsymbol{H}_{0}(\operatorname{rot}, \Omega):=\left\{\boldsymbol{q} \in \boldsymbol{L}^{2}(\Omega) \mid \operatorname{rot} \boldsymbol{q} \in L^{2}(\Omega), \boldsymbol{q} \cdot \boldsymbol{\tau}=0 \text { on } \partial \Omega\right\}
$$

with $\boldsymbol{\tau}$ the unit tangent to $\partial \Omega$, and $\operatorname{rot} \boldsymbol{q}=\operatorname{rot}\left(q_{1}, q_{2}\right)=\partial_{x} q_{2}-\partial_{y} q_{1}$. It can be shown that

$$
\boldsymbol{H}^{-1}(\operatorname{div}, \Omega)=\left\{\boldsymbol{q} \in \boldsymbol{H}^{-1}(\Omega) \mid \operatorname{div} \boldsymbol{q} \in H^{-1}(\Omega)\right\}
$$

with $\operatorname{div} \boldsymbol{q}:=\partial_{x} q_{1}+\partial_{y} q_{2}$. Define

$$
\boldsymbol{H}(\operatorname{div}, \Omega):=\left\{\boldsymbol{q} \in \boldsymbol{L}^{2}(\Omega) \mid \operatorname{div} \boldsymbol{q} \in L^{2}(\Omega)\right\},
$$

and the norm in $\boldsymbol{H}(\operatorname{div}, \Omega)$ is given by

$$
\|\boldsymbol{q}\|_{\boldsymbol{H}(\mathrm{div})}:=\left(\|\boldsymbol{q}\|_{0}^{2}+\|\operatorname{div} \boldsymbol{q}\|_{0}^{2}\right)^{1 / 2} .
$$

We recast (2.1) and (2.2) into a mixed variational problem as follows.

Problem 2.1. Find $(\phi, \omega, \gamma) \in \boldsymbol{V} \times W \times \boldsymbol{M}$ such that

$$
\mathcal{A}(\boldsymbol{\phi}, \omega, \boldsymbol{\gamma} ; \boldsymbol{\psi}, v, \boldsymbol{s})=(g, v) \quad \forall(\boldsymbol{\psi}, v, \boldsymbol{s}) \in \boldsymbol{V} \times W \times \boldsymbol{M},
$$

where

$$
\mathcal{A}(\boldsymbol{\phi}, \omega, \boldsymbol{\gamma} ; \boldsymbol{\psi}, v, \boldsymbol{s}):=a(\boldsymbol{\phi}, \boldsymbol{\psi})+(\boldsymbol{\gamma}, \nabla v-\boldsymbol{\psi})+(\nabla \omega-\boldsymbol{\phi}, \boldsymbol{s})-\lambda^{-1} t^{2}(\boldsymbol{\gamma}, \boldsymbol{s}) .
$$

The following a priori estimates and refined regularity properties are included in [5, 15, 26, and in particular [28, Theorem 2.1],

$$
\begin{aligned}
&\|\omega\|_{1}+\|\phi\|_{1}+\|\gamma\|_{0} \leq C\|g\|_{-1}, \\
&\|\phi\|_{2} \leq C\|g\|_{-1}, \quad\|\omega\|_{2} \leq C\left(\|g\|_{-1}+t^{2}\|g\|_{0}\right), \\
&\|\operatorname{div} \gamma\|_{0} \leq C\|g\|_{0}, \quad t\|\gamma\|_{1} \leq C\left(\|g\|_{-1}+t\|g\|_{0}\right) .
\end{aligned}
$$

The following regularity estimate is crucial for the $\mathrm{L}^{2}$ error estimate of the stabilized MITC4 element.

Lemma 2.2. Let $\phi$ be solution of (2.1); then

$$
\|\operatorname{rot} \phi\|_{0} \leq C t\|g\|_{-1} \text {. }
$$

Proof. Following the Appendix of [5], we obtain

$$
\gamma=\nabla r+\operatorname{curl} p
$$

where $r$ and $p$ are solutions of some auxiliary problems. Using the definition of $\gamma$ (2.2), we get $\operatorname{rot} \phi=-\lambda^{-1} t^{2} \operatorname{rot} \gamma$, which together with the above identity leads to

$$
\operatorname{rot} \phi=\lambda^{-1} t^{2} \triangle p \text {. }
$$

We thus have

$$
\|\operatorname{rot} \phi\|_{0} \leq C t^{2}|p|_{2} \leq C t\|g\|_{-1},
$$

where we have used $t\|p\|_{2} \leq C\|g\|_{-1}$ (see, e.g., [5, inequality (7.5)]).

Remark 2.3. If there is an extra forcing term $(\boldsymbol{F}, \boldsymbol{\psi})$ in the right-hand side of (2.1), then the estimate (2.7) changes to

$$
\|\operatorname{rot} \phi\|_{0} \leq C t\left(\|g\|_{-1}+\|\boldsymbol{F}\|_{0}\right) .
$$

The proof follows the same line of Lemma 2.2 . 


\section{Finite ELEMENT APPROXIMATION}

Let $\mathcal{C}_{h}$ be a partition of $\bar{\Omega}$ by convex quadrilaterals $K$ with diameter $h_{K}$, and the subscript $h$ is defined by $h=\max _{K \in \mathcal{C}_{h}} h_{K}$. We assume that $\mathcal{C}_{h}$ is shape regular in the sense of Ciarlet-Raviart (see [16, p. 247]). Define $\mathcal{P}_{k}$ as the space of polynomials of degree no more than $k, \mathcal{Q}_{m, n}$ the space of polynomials of degree no more than $m$ in the first variable and $n$ in the second variable, and denote $\mathcal{Q}_{k}=\mathcal{Q}_{k, k}$ for brevity.

Let $\hat{K}=(-1,1)^{2}$ be the reference square. For each element $K \in \mathcal{C}_{h}$, there exists a bilinear map $\boldsymbol{F}$ such that $\boldsymbol{F}(\hat{K})=K$ with

$$
\boldsymbol{F}=\left(a_{0}+a_{1} \hat{x}+a_{2} \hat{y}+a_{12} \hat{x} \hat{y}, b_{0}+b_{1} \hat{x}+b_{2} \hat{y}+b_{12} \hat{x} \hat{y}\right) .
$$

In view of the left figure in Figure 1, we have $\overrightarrow{M_{4} M_{2}}=2\left(a_{1}, b_{1}\right), \overrightarrow{M_{1} M_{3}}=2\left(a_{2}, b_{2}\right)$ and $\overrightarrow{O_{1} O_{2}}=2\left(a_{12}, b_{12}\right)$. An elementary calculation gives $\left|a_{1}\right|,\left|a_{2}\right|,\left|a_{12}\right|,\left|b_{1}\right|,\left|b_{2}\right|$, $\left|b_{12}\right| \leq h_{K}$.

To each scalar function $\hat{v}$ defined on $\hat{K}$, we associate it with a function $v$ defined on $K$ such that $v(\boldsymbol{x})=v(\boldsymbol{F}(\hat{\boldsymbol{x}}))=\hat{v}(\hat{\boldsymbol{x}})$. Given a vector function $\hat{\boldsymbol{v}}$, we define $\boldsymbol{v}$ on $K$ by the rotated Piola transform as

$$
\boldsymbol{v}(\boldsymbol{x})=[D \boldsymbol{F}(\hat{\boldsymbol{x}})]^{-T} \hat{\boldsymbol{v}}(\hat{\boldsymbol{x}}),
$$

where $\boldsymbol{x}=\boldsymbol{F}(\hat{\boldsymbol{x}})$, and $D \boldsymbol{F}(\hat{\boldsymbol{x}})$ is the Jacobian matrix of the mapping $\boldsymbol{F} . A^{T}$ denotes the transpose of a matrix $A$.

We introduce below a mesh condition that quantifies the deviation of a quadrilateral from a parallelogram.

$(1+\alpha)$-Section Condition $(0 \leq \alpha \leq 1)$ 31]. The distance $d_{K}$ between the midpoints of two diagonals of $K$ is $\mathcal{O}\left(h_{K}^{1+\alpha}\right)$ uniformly for all elements $K \in \mathcal{C}_{h}$ as $h \rightarrow 0$.

The extreme case $\alpha=0$ represents an unstructured quadrilateral mesh subdivision. The mesh in the right figure of Figure 1 is a particular one, which consists of trapezoids generating from a typical trapezoid with translation and dilation. In the case of $\alpha=1$, the mesh satisfies the Bi-Section Condition [34. We call a mesh an asymptotically regular parallelogram mesh if it is shape regular and satisfies the Bi-Section Condition simultaneously.
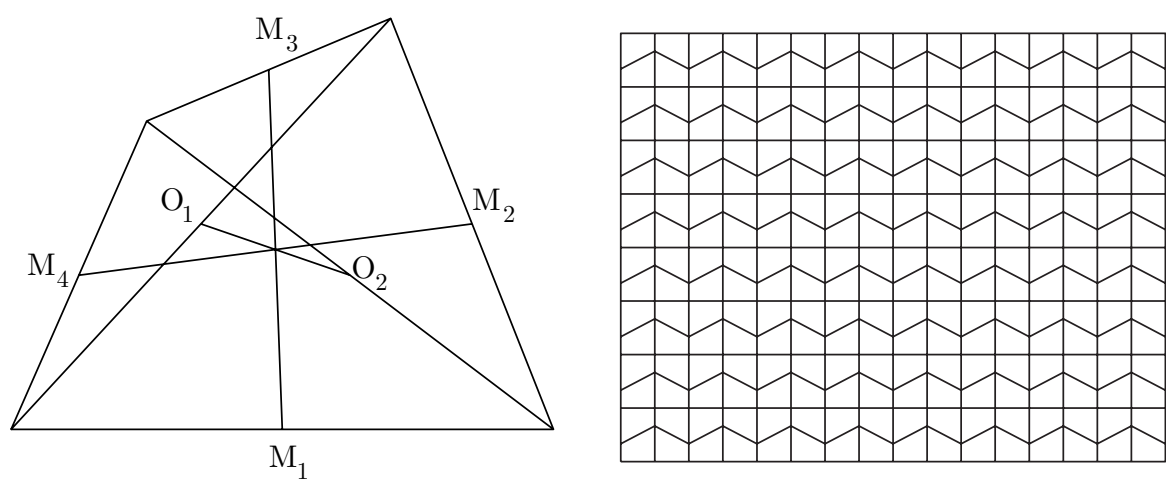

Figure 1. Left: A typical quadrilateral. Right: A particular mesh satisfies $\alpha=0$. 
Remark 3.1. In 22, the authors call the $(1+\alpha)$-Section Condition the $(1+\alpha)$ Regular Condition.

Define the finite element approximation spaces as

$$
\begin{aligned}
& \boldsymbol{V}_{h}:=\left\{\boldsymbol{\psi} \in \boldsymbol{V} \mid \boldsymbol{\psi}_{\left.\right|_{K}} \in\left[\mathcal{Q}_{1}(K)\right]^{2} \quad \forall K \in \mathcal{C}_{h}\right\}, \\
& W_{h}:=\left\{v \in W \mid v_{\left.\right|_{K}} \in \mathcal{Q}_{1}(K) \quad \forall K \in \mathcal{C}_{h}\right\} .
\end{aligned}
$$

Define $\Pi_{1}:=\left(\Pi_{1}, \Pi_{1}\right)$, where $\Pi_{1}$ is the standard bilinear interpolation operator.

We define two kinds of $\boldsymbol{H}_{0}$ (rot) finite element spaces. One is constructed by Thomas [36] as

$$
\boldsymbol{\Gamma}_{h}:=\left\{\boldsymbol{s} \in \boldsymbol{H}_{0}(\operatorname{rot}, \Omega) \cap \boldsymbol{H}^{1}(\Omega) \mid \boldsymbol{s}=D \boldsymbol{F}^{-T} \hat{\boldsymbol{s}}, \hat{\boldsymbol{s}} \in \boldsymbol{\Gamma}(\hat{K}) \quad \forall K \in \mathcal{C}_{h}\right\},
$$

where $\boldsymbol{\Gamma}(\hat{K})$ is spanned by $(1,0),(0,1),(\hat{y}, 0)$ and $(0, \hat{x})$. The standard interpolation operator into $\boldsymbol{\Gamma}_{h}$ is denoted by $\Pi$ (see [36] for a definition).

Another one has been proposed recently by Arnold, Boffi and Falk [2] $\left(A B F_{0}\right)$. It is the same as $\boldsymbol{\Gamma}_{h}$ except that $\boldsymbol{\Gamma}(\hat{K})$ is spanned by $(1,0),(0,1),(\hat{y}, 0),(0, \hat{x}),\left(\hat{y}^{2}, 0\right)$ and $\left(0, \hat{x}^{2}\right)$. The standard interpolation operator into this space is denoted by $\widetilde{\Pi}$ (see [2] for details).

We denote $\Pi$ and $\widetilde{\Pi}$ as $\boldsymbol{R}_{h}$, and let $\boldsymbol{R}_{h_{\left.\right|_{K}}}=\boldsymbol{R}_{K}, \Pi_{\left.\right|_{K}}=\Pi_{K}$ and $\widetilde{\Pi}_{\left.\right|_{K}}=\widetilde{\Pi}_{K}$.

3.1. The stabilized MITC4 element. The stabilized MITC4 element (ixed Interpolation for Tensorial Components $\underline{4}$-nodes) was introduced by Lyly, Stenberg and Vihinen 27. The main ingredient of this element is the MITC interpolation operator $\Pi$.

The method is realized through the following bilinear form $\mathcal{B}_{h}$ and the linear form $l_{h}$. For any $(\boldsymbol{\eta}, w)$ and $(\boldsymbol{\psi}, v)$ in $\boldsymbol{V}_{h} \times W_{h}$, we define $\mathcal{B}_{h}$ as

$$
\mathcal{B}_{h}(\boldsymbol{\eta}, w ; \boldsymbol{\psi}, v):=a(\boldsymbol{\eta}, \boldsymbol{\psi})+\sum_{K \in \mathcal{C}_{h}} \frac{\lambda}{t^{2}+\alpha_{K} h_{K}^{2}}\left(\nabla w-\Pi_{K} \boldsymbol{\eta}, \nabla v-\Pi_{K} \boldsymbol{\psi}\right),
$$

and $\ell_{h}(\boldsymbol{\psi}, v)=(g, v)$, where $\alpha_{K}$ is a piecewise constant. The method is formulated as: find $\left(\phi_{h}, \omega_{h}\right) \in V_{h} \times W_{h}$ such that

$$
\mathcal{B}_{h}\left(\phi_{h}, \omega_{h} ; \boldsymbol{\psi}, v\right)=\ell_{h}(\boldsymbol{\psi}, v) \quad \forall(\boldsymbol{\psi}, v) \in \boldsymbol{V}_{h} \times W_{h} .
$$

Given $\left(\phi_{h}, \omega_{h}\right)$, the solution of the above equation, $\gamma_{h}$ is locally defined as

$$
\gamma_{h_{K}}:=\frac{\lambda}{t^{2}+\alpha_{K} h_{K}^{2}}\left(\nabla \omega_{h}-\Pi_{K} \phi_{h}\right) \quad \forall K \in \mathcal{C}_{h} .
$$

Remark 3.2. If we set $\alpha_{K}=0$, the stabilized MITC4 element reduces to the MITC4 element of Bathe and Dvorkin [11.

Remark 3.3. There is another kind of stabilized MITC4 element which is the lowest order case of the general MITC stabilized method in [26, Method 4.1 with $k=1$ ]. As shown in [30, all results for the stabilized MITC4 element defined above also hold true for that case.

3.2. MIN4 element. The MIN4 element (Mindlin element 4 nodes) was introduced by Tessler and Hughes [35], which employed two types of the numerical stabilization tricks.

The deflection is first approximated by the serendipity quadratic element $\mathcal{Q}_{2}^{r}$, which can be locally decomposed as $\mathcal{Q}_{2}^{r}(K)=\mathcal{Q}_{1}(K)+\mathcal{E}(K)$, where

$$
\mathcal{E}(K)=\left\{v \in \mathcal{Q}_{2}^{r}(K) \mid v=0 \quad \text { at all vertices of } K\right\} .
$$


Second, the part of the deflection belonging to $\mathcal{E}(K)$ is eliminated by forcing the tangent component of the discrete shear strain to be constant along element edges. Let $\mathcal{L}_{K}:\left[\mathcal{Q}_{1}(K)\right]^{2} \rightarrow \mathcal{E}(K)$ be the linear operator defined by

$$
\frac{\partial}{\partial \boldsymbol{s}}\left(\left(\nabla \mathcal{L}_{K} \boldsymbol{\psi}-\boldsymbol{\psi}\right) \cdot \boldsymbol{\tau}\right)=0 \quad \text { along each edge of } K
$$

with $s$ the arc-length. The operator $\mathcal{L}_{K}$ is called the kinematically linked interpolation operator.

Another numerical stabilization trick is the so-called modified shear correction factor which emanated from Fried and Young's work [18. When isotropic materials are taken into account, Tessler and Hughes proposed that the numerical factor $t^{2} /\left(t^{2}+\alpha_{K} h_{K}^{2}\right)$ should be used to locally balance the shear strain energy term, where $\alpha_{K}$ is a positive parameter independent of $t$ and $h$.

For any $(\boldsymbol{\eta}, w)$ and $(\boldsymbol{\psi}, v)$ in $\boldsymbol{V}_{h} \times W_{h}, \mathcal{B}_{h}$ and $\ell_{h}$ are defined as

$\mathcal{B}_{h}(\boldsymbol{\eta}, w ; \boldsymbol{\psi}, v):=a(\boldsymbol{\eta}, \boldsymbol{\psi})+\sum_{K \in \mathcal{C}_{h}} \frac{\lambda}{t^{2}+\alpha_{K} h_{K}^{2}}\left(\nabla\left(w+\mathcal{L}_{K} \boldsymbol{\eta}\right)-\boldsymbol{\eta}, \nabla\left(v+\mathcal{L}_{K} \boldsymbol{\psi}\right)-\boldsymbol{\psi}\right)$

and $\ell_{h}(\boldsymbol{\psi}, v):=\sum_{K \in \mathcal{C}_{h}}\left(g, v+\mathcal{L}_{K} \boldsymbol{\psi}\right)$.

The MIN4 element is defined as: find $\left(\phi_{h}, \omega_{h}\right) \in \boldsymbol{V}_{h} \times W_{h}$ such that

$$
\mathcal{B}_{h}\left(\phi_{h}, \omega_{h} ; \boldsymbol{\psi}, v\right)=\ell_{h}(\boldsymbol{\psi}, v) \quad \forall(\boldsymbol{\psi}, v) \in \boldsymbol{V}_{h} \times W_{h},
$$

and the shear stress is computed from

$$
\gamma_{\left.h\right|_{K}}:=\frac{\lambda}{t^{2}+\alpha_{K} h_{K}^{2}}\left(\nabla\left(\omega_{h}+\mathcal{L}_{K} \phi_{h}\right)-\phi_{h}\right) .
$$

3.3. Q4BL element. The third method to be considered is the Q4BL element (Quadrilateral $\underline{4}$ nodes Bubble Stabilized Linked interpolated), which was introduced by Zienkiewicz, Xu, Zeng, Samuelsson and Wiberg [39]. The stabilization trick used in this element is similar to that in the MIN4 element.

The main difference between the MIN4 element and the Q4BL element is that the latter is based upon a mixed formulation and uses a bubble-enriched space for approximating the rotations. The finite element space for the rotations is defined as

$$
\boldsymbol{V}_{h}^{*}:=\boldsymbol{V}_{h}+\boldsymbol{V}_{h}^{0}
$$

with the quadratic bubble function space

$$
\boldsymbol{V}_{h}^{0}:=\left\{\boldsymbol{\psi} \in \boldsymbol{V} \mid \boldsymbol{\psi}_{\left.\right|_{K}} \in \mathcal{B}(K) \quad \forall K \in \mathcal{C}_{h}\right\},
$$

where $\mathcal{B}(K):=\operatorname{Span}\left[\left(1-\hat{x}^{2}\right)\left(1-\hat{y}^{2}\right)\right]^{2}$. The approximation procedure for the deflection is still first approximated by the serendipity quadratic element $\mathcal{Q}_{2}^{r}$, and then the part of $\mathcal{E}(K)$ is eliminated by forcing the tangent component of the discrete shear strain to be constant along element edges. The shear stress is approximated by a piecewise constant element as

$$
\boldsymbol{M}_{h}:=\left\{s \in \boldsymbol{s} \mid \boldsymbol{s}_{\left.\right|_{K}} \in\left[\mathcal{P}_{0}(K)\right]^{2} \quad \forall K \in \mathcal{C}_{h}\right\} .
$$

The method is formulated as: find $\left(\phi_{h}, \omega_{h}, \gamma_{h}\right) \in \boldsymbol{V}_{h}^{*} \times W_{h} \times \boldsymbol{M}_{h}$ such that

$$
\begin{array}{r}
a\left(\boldsymbol{\phi}_{h}, \boldsymbol{\psi}\right)+\sum_{K \in \mathcal{C}_{h}}\left(\gamma_{h}, \nabla\left(v+\mathcal{L}_{K} \boldsymbol{\psi}\right)-\boldsymbol{\psi}\right)=l_{h}(v, \psi) \quad \forall(\boldsymbol{\psi}, v) \in \boldsymbol{V}_{h}^{*} \times W_{h}, \\
\sum_{K \in \mathcal{C}_{h}}\left(\nabla\left(\omega_{h}+\mathcal{L}_{K} \boldsymbol{\phi}_{h}\right)-\boldsymbol{\phi}_{h}, \boldsymbol{s}\right)-\lambda^{-1} t^{2}\left(\boldsymbol{\gamma}_{h}, \boldsymbol{s}\right)=0 \quad \forall \boldsymbol{s} \in \boldsymbol{M}_{h},
\end{array}
$$


where $\ell_{h}(v, \boldsymbol{\psi}):=\sum_{K \in \mathcal{C}_{h}}\left(g, v+\mathcal{L}_{K} \boldsymbol{\psi}\right)$.

Eliminating the shear stress $\gamma_{h}$ on the element level from (3.5), we obtain

$$
\gamma_{\left.h\right|_{K}}=\lambda t^{-2} \boldsymbol{\Pi}_{0}\left(\nabla\left(\omega_{h}+\mathcal{L}_{K} \phi_{h}\right)-\phi_{h}\right),
$$

where $\Pi_{0}=\left(\Pi_{0}, \Pi_{0}\right)$ with $\Pi_{0}$ the $L^{2}$ projection operator onto $\mathcal{P}_{0}(K)$. Substituting (3.6) into (3.4), we get the following displacement oriented variational problem: find $\left(\phi_{h}, \omega_{h}\right) \in \boldsymbol{V}_{h} \times W_{h}$ such that for all $(\boldsymbol{\psi}, v) \in \boldsymbol{V}_{h} \times W_{h}$

$a\left(\phi_{h}, \boldsymbol{\psi}\right)+\lambda t^{-2} \sum_{K \in \mathcal{C}_{h}}\left(\boldsymbol{\Pi}_{0}\left(\nabla\left(\omega_{h}+\mathcal{L}_{K} \boldsymbol{\phi}_{h}\right)-\boldsymbol{\phi}_{h}\right), \boldsymbol{\Pi}_{0}\left(\nabla\left(v+\mathcal{L}_{K} \boldsymbol{\psi}\right)-\boldsymbol{\psi}\right)\right)=\ell_{h}(v, \boldsymbol{\psi})$.

3.4. FMIN4 element. The element proposed by Kikuchi and Ishii (Full MIN4) 21] is similar to the MIN4 element. The only difference is that it employs the complete quadratic element $\mathcal{Q}_{2}$ for the deflection and the constraints in the definition of $\mathcal{L}_{K}$ are imposed not only on the four edges but also the two centerlines, and we denote by $\widetilde{\mathcal{L}}_{K}$ this full kinematically linked interpolation operator. The numerical shear correction factor is also used to balance the shear strain energy.

Remark 3.4. The employment of the full $\mathcal{Q}_{2}$ in the FMIN4 element is to account for the mesh distortion, which is justified by our later theoretic results; cf. Theorems 5.5 and 5.7 (see also [3] for related discussions.)

\section{Connections between the elements}

In this section, we consider the connection between the elements discussed above. The key ingredient is an identity connecting the kinematically linked interpolation operator $\mathcal{L}_{K}, \widetilde{\mathcal{L}}_{K}$ and the Raviart-Thomas type interpolation operator $\Pi_{K}, \widetilde{\Pi}_{K}$, which will be presented in the first part of this section. Some properties of $\mathcal{L}_{K}$ and $\widetilde{\mathcal{L}}_{K}$ are given in the second part. We shall reformulate all elements in the last part.

4.1. Identities for $\mathcal{L}_{K}$ and $\widetilde{\mathcal{L}}_{K}$. As proven in [24, Theorem 4.1], there is a beautiful identity between the gradient of $\mathcal{L}_{K}$ and $\Pi_{K}$ for the linear triangular element, i.e.,

$$
\nabla \mathcal{L}_{K} \boldsymbol{\psi}=\boldsymbol{\psi}-\Pi_{K} \boldsymbol{\psi} \quad \forall \boldsymbol{\psi} \in\left[\mathcal{P}_{1}(K)\right]^{2} .
$$

Unfortunately, this identity does not hold when $\psi$ belongs to $\left[\mathcal{Q}_{1}(K)\right]^{2}$; the method exploited in $\left[24\right.$ is not applicable in the present situation since $\left[\mathcal{Q}_{1}(K)\right]^{2}$ cannot be hierarchically decomposed into $\boldsymbol{\Gamma}(K)+\nabla \mathcal{E}(K)$. Nevertheless, in the next theorem, we shall prove a similar identity for $\mathcal{L}_{K}$ with a residue term. As for $\widetilde{\mathcal{L}}_{K}$, we shall establish an identity in Theorem 4.2 as

$$
\nabla \widetilde{\mathcal{L}}_{K} \boldsymbol{\psi}=\boldsymbol{\psi}-\widetilde{\Pi}_{K} \boldsymbol{\psi} \quad \forall \boldsymbol{\psi} \in\left[\mathcal{Q}_{1}(K)\right]^{2} .
$$

Naturally, this identity can be viewed as a quadrilateral analog of (4.1).

\section{Theorem 4.1.}

$$
\nabla \mathcal{L}_{K} \boldsymbol{\psi}=\boldsymbol{\psi}-\Pi_{K} \boldsymbol{\psi}+\boldsymbol{\psi}^{*} \quad \forall \boldsymbol{\psi} \in\left[\mathcal{Q}_{1}(K)\right]^{2} .
$$

The residue $\boldsymbol{\psi}^{*}$ may be written into $\boldsymbol{\psi}^{*}=\boldsymbol{\psi}_{1}^{*}+\boldsymbol{\psi}_{2}^{*}$ with

$$
\begin{aligned}
\left|\left(\boldsymbol{s}, \boldsymbol{\psi}_{1}^{*}\right)_{K}\right| & \leq C h_{K}^{2}\|\operatorname{div} \boldsymbol{s}\|_{0, K}|\boldsymbol{\psi}|_{1, K} \quad \forall \boldsymbol{s} \in \boldsymbol{H}(\operatorname{div}, K), \\
\left\|\boldsymbol{\psi}_{2}^{*}\right\|_{0, K} & \leq C h_{K}\left\|\operatorname{rot}\left(\boldsymbol{\psi}-\Pi_{K} \boldsymbol{\psi}\right)\right\|_{0, K} .
\end{aligned}
$$


Proof. Our proof is based on a direct calculation.

In view of the definition of $\Pi_{K}$, we obtain

$$
\boldsymbol{\psi}-\Pi_{K} \boldsymbol{\psi}=D \boldsymbol{F}^{-T}\left(D \boldsymbol{F}^{T} \boldsymbol{\psi}-\Pi_{K} D \boldsymbol{F}^{T} \boldsymbol{\psi}\right) .
$$

On the reference element $\hat{K}$, we write $\boldsymbol{\psi}$ as $\boldsymbol{\psi}(\boldsymbol{x})=\widehat{\boldsymbol{\psi}}(\hat{\boldsymbol{x}})=\left(\widehat{\psi}_{1}, \widehat{\psi}_{2}\right)^{T}$ with $\widehat{\psi}_{1}(\hat{x}, \hat{y})=A_{0}+A_{1} \hat{x}+A_{2} \hat{y}+A_{12} \hat{x} \hat{y} \quad$ and $\quad \widehat{\psi}_{2}(\hat{x}, \hat{y})=B_{0}+B_{1} \hat{x}+B_{2} \hat{y}+B_{12} \hat{x} \hat{y}$, where

$$
\begin{aligned}
& A_{0}=\widehat{\psi}_{1}(0,0), \quad A_{1}=\frac{\partial \widehat{\psi}_{1}}{\partial \hat{x}}(0,0), \quad A_{2}=\frac{\partial \widehat{\psi}_{1}}{\partial \hat{y}}(0,0), \quad A_{12}=\frac{\partial^{2} \widehat{\psi}_{1}}{\partial \hat{x} \partial \hat{y}}, \\
& B_{0}=\widehat{\psi}_{2}(0,0), \quad B_{1}=\frac{\partial \widehat{\psi}_{2}}{\partial \hat{x}}(0,0), \quad B_{2}=\frac{\partial \widehat{\psi}_{2}}{\partial \hat{y}}(0,0), \quad B_{12}=\frac{\partial^{2} \widehat{\psi}_{2}}{\partial \hat{x} \partial \hat{y}} .
\end{aligned}
$$

Invoking (3.1) and the above expression, we get

$$
\begin{aligned}
\left(D \boldsymbol{F}^{T} \boldsymbol{\psi}-\Pi_{K} D \boldsymbol{F}^{T} \boldsymbol{\psi}\right)_{1} & =\left(a_{1} A_{1}+b_{1} B_{1}\right) \hat{x}+\left(a_{1} A_{12}+a_{12} A_{1}+b_{1} B_{12}+b_{12} B_{1}\right) \hat{x} \hat{y} \\
& +\left(a_{12} A_{12}+b_{12} B_{12}\right) \hat{x} \hat{y}^{2}-\left(a_{12} A_{2}+b_{12} B_{2}\right)\left(1-\hat{y}^{2}\right), \\
\left(D \boldsymbol{F}^{T} \boldsymbol{\psi}-\Pi_{K} D \boldsymbol{F}^{T} \boldsymbol{\psi}\right)_{2} & =\left(a_{2} A_{2}+b_{2} B_{2}\right) \hat{y}+\left(a_{2} A_{12}+a_{12} A_{2}+b_{2} B_{12}+b_{12} B_{2}\right) \hat{x} \hat{y} \\
& +\left(a_{12} A_{12}+b_{12} B_{12}\right) \hat{x}^{2} \hat{y}-\left(a_{12} A_{1}+b_{12} B_{1}\right)\left(1-\hat{x}^{2}\right) .
\end{aligned}
$$

Noting $\nabla \mathcal{L}_{K} \boldsymbol{\psi}=D \boldsymbol{F}^{-T} \hat{\nabla} \widehat{\mathcal{L}_{K} \boldsymbol{\psi}}$ and using the explicit expression in [35, we have

$$
\begin{aligned}
\frac{\partial}{\partial \hat{x}} \widehat{\mathcal{L}_{K} \psi}= & \left(a_{1} A_{1}+b_{1} B_{1}+a_{12} A_{12}+b_{12} B_{12}\right) \hat{x} \\
& +\left(a_{1} A_{12}+a_{12} A_{1}+b_{1} B_{12}+b_{12} B_{1}\right) \hat{x} \hat{y} \\
& -\frac{1}{2}\left(a_{12} A_{2}+a_{2} A_{12}+b_{12} B_{2}+b_{2} B_{12}\right)\left(1-\hat{y}^{2}\right), \\
\frac{\partial}{\partial \hat{y}} \widehat{\mathcal{L}_{K} \psi}= & \left(a_{2} A_{2}+b_{2} B_{2}+a_{12} A_{12}+b_{12} B_{12}\right) \hat{y} \\
& +\left(a_{2} A_{12}+a_{12} A_{2}+b_{2} B_{12}+b_{12} B_{2}\right) \hat{x} \hat{y} \\
& -\frac{1}{2}\left(a_{12} A_{1}+a_{1} A_{12}+b_{12} B_{1}+b_{1} B_{12}\right)\left(1-\hat{x}^{2}\right) .
\end{aligned}
$$

A combination of the four equations above yields (4.2). The residue $\boldsymbol{\psi}^{*}$ can be further decomposed into $\boldsymbol{\psi}^{*}=\boldsymbol{\psi}_{1}^{*}+\boldsymbol{\psi}_{2}^{*}$ with

$$
\begin{aligned}
& \boldsymbol{\psi}_{1}^{*}:=D \boldsymbol{F}^{-T}\left(a_{12} A_{12}+b_{12} B_{12}\right)\left(\hat{x}\left(1-\hat{y}^{2}\right), \hat{y}\left(1-\hat{x}^{2}\right)\right)^{T}, \\
& \boldsymbol{\psi}_{2}^{*}:=D \boldsymbol{F}^{-T}\left(\mathcal{C}\left(1-\hat{y}^{2}\right) / 2, \mathcal{D}\left(1-\hat{x}^{2}\right) / 2\right)^{T},
\end{aligned}
$$

where $\mathcal{C}:=a_{12} A_{2}+b_{12} B_{2}-a_{2} A_{12}-b_{2} B_{12}$ and $\mathcal{D}:=a_{12} A_{1}+b_{12} B_{1}-a_{1} A_{12}-b_{1} B_{12}$.

Note that $\boldsymbol{\psi}_{1}^{*}=\nabla q$ with $q=-\frac{1}{2}\left(a_{12} A_{12}+b_{12} B_{12}\right)\left(\hat{x}^{2}-1\right)\left(\hat{y}^{2}-1\right)$. For any $\boldsymbol{s} \in \boldsymbol{H}(\operatorname{div}, K)$, an integration by parts leads to

$$
\left(\boldsymbol{s}, \boldsymbol{\psi}_{1}^{*}\right)_{K}=(\boldsymbol{s}, \nabla q)_{K}=-(\operatorname{div} \boldsymbol{s}, q)_{K}
$$

since $q \in H_{0}^{1}(K)$. Using Poincaré's inequality, we obtain

$$
\|q\|_{0, K} \leq C h_{K}\|\nabla q\|_{0, K} .
$$

Note that $A_{12}$ and $B_{12}$ can be rewritten into

$$
A_{12}=\frac{\partial \widehat{\psi}_{1}}{\partial \hat{x}}(0,1)-\frac{\partial \widehat{\psi}_{1}}{\partial \hat{x}}(0,0), \quad B_{12}=\frac{\partial \widehat{\psi}_{2}}{\partial \hat{x}}(0,1)-\frac{\partial \widehat{\psi}_{2}}{\partial \hat{x}}(0,0) ;
$$


we thus have

$$
\left\|\boldsymbol{\psi}_{1}^{*}\right\|_{0, K} \leq C\left|a_{12} A_{12}+b_{12} B_{12}\right| \leq C h_{K}|\widehat{\boldsymbol{\psi}}|_{1, \hat{K}} \leq C h_{K}|\boldsymbol{\psi}|_{1, K} .
$$

Combining the estimate for $q$ and (4.8) leads to

$$
\begin{aligned}
\left|\left(\boldsymbol{s}, \boldsymbol{\psi}_{1}^{*}\right)_{K}\right| & \leq\|\operatorname{div} \boldsymbol{s}\|_{0, K}\|q\|_{0, K} \leq C h_{K}\|\operatorname{div} \boldsymbol{s}\|_{0, K}\|\nabla q\|_{0, K} \\
& =C h_{K}\|\operatorname{div} \boldsymbol{s}\|_{0, K}\left\|\boldsymbol{\psi}_{1}^{*}\right\|_{0, K} \leq C h_{K}^{2}\|\operatorname{div} \boldsymbol{s}\|_{0, K}|\boldsymbol{\psi}|_{1, K},
\end{aligned}
$$

which gives $(4.3){ }_{1}$.

Using (4.7), we get $\left\|\boldsymbol{\psi}_{2}^{*}\right\|_{0, \hat{K}}^{2}=\frac{8}{15}\left(\mathcal{C}^{2}+\mathcal{D}^{2}\right)=\frac{4}{3}\left\|\widehat{\operatorname{rot}}_{\boldsymbol{\psi}_{2}}^{*}\right\|_{0, \hat{K}}$. Noting that $\operatorname{rot} \boldsymbol{\psi}_{2}^{*}=\operatorname{rot}\left(\Pi_{K} \boldsymbol{\psi}-\boldsymbol{\psi}\right)$, we get

$$
\begin{aligned}
\left\|\boldsymbol{\psi}_{2}^{*}\right\|_{0, K} & \leq C\left\|\widehat{\boldsymbol{\psi}}_{2}^{*}\right\|_{0, \hat{K}}=\frac{4}{3} C\left\|\widehat{\operatorname{rot}} \widehat{\boldsymbol{\psi}}_{2}^{*}\right\|_{0, \hat{K}} \leq C h_{K}\left\|\operatorname{rot} \boldsymbol{\psi}_{2}^{*}\right\|_{0, K} \\
& =C h_{K}\left\|\operatorname{rot}\left(\boldsymbol{\psi}-\Pi_{K} \boldsymbol{\psi}\right)\right\|_{0, K},
\end{aligned}
$$

which yields $(4.3)_{2}$.

\section{Theorem 4.2.}

$$
\nabla \widetilde{\mathcal{L}}_{K} \boldsymbol{\psi}=\boldsymbol{\psi}-\widetilde{\Pi}_{K} \boldsymbol{\psi} \quad \forall \boldsymbol{\psi} \in\left[\mathcal{Q}_{1}(K)\right]^{2} .
$$

Proof. Proceeding along the same line as Theorem 4.1 noting that

$$
\begin{aligned}
\left(D \boldsymbol{F}^{T} \boldsymbol{\psi}-\widetilde{\Pi}_{K} D \boldsymbol{F}^{T} \boldsymbol{\psi}\right)_{1}= & \left(D \boldsymbol{F}^{T} \boldsymbol{\psi}-\Pi_{\hat{K}} D \boldsymbol{F}^{T} \boldsymbol{\psi}\right)_{1} \\
& +\left(a_{12} A_{2}+b_{12} B_{2}-a_{2} A_{12}-b_{2} B_{12}\right)\left(1-\hat{y}^{2}\right) / 2, \\
\left(D \boldsymbol{F}^{T} \boldsymbol{\psi}-\widetilde{\Pi}_{K} D \boldsymbol{F}^{T} \boldsymbol{\psi}\right)_{2}= & \left(D \boldsymbol{F}^{T} \boldsymbol{\psi}-\Pi_{\hat{K}} D \boldsymbol{F}^{T} \boldsymbol{\psi}\right)_{2} \\
& +\left(a_{12} A_{1}+b_{12} B_{1}-a_{1} A_{12}-b_{1} B_{12}\right)\left(1-\hat{x}^{2}\right) / 2,
\end{aligned}
$$

and using the explicit expression of the gradient of $\widetilde{\mathcal{L}}_{K}$ [21],

$$
\begin{aligned}
\frac{\partial}{\partial \hat{x}} \widehat{\widetilde{\mathcal{L}}_{K} \boldsymbol{\psi}}=\left(a_{1} A_{1}+b_{1} B_{1}\right) \hat{x}+\left(a_{1} A_{12}+a_{12} A_{1}+b_{1} B_{12}+b_{12} B_{1}\right) \hat{x} \hat{y} \\
-\frac{1}{2}\left(a_{12} A_{2}+a_{2} A_{12}+b_{12} B_{2}+b_{2} B_{12}\right)\left(1-\hat{y}^{2}\right)+\left(a_{12} A_{12}+b_{12} B_{12}\right) \hat{x} \hat{y}^{2}, \\
\frac{\partial}{\partial \hat{y}} \widehat{\widetilde{\mathcal{L}}_{K} \boldsymbol{\psi}}=\left(a_{2} A_{2}+b_{2} B_{2}\right) \hat{y}+\left(a_{2} A_{12}+a_{12} A_{2}+b_{2} B_{12}+b_{12} B_{2}\right) \hat{x} \hat{y} \\
-\frac{1}{2}\left(a_{12} A_{1}+a_{1} A_{12}+b_{12} B_{1}+b_{1} B_{12}\right)\left(1-\hat{x}^{2}\right)+\left(a_{12} A_{12}+b_{12} B_{12}\right) \hat{x}^{2} \hat{y}
\end{aligned}
$$

we come to 4.11).

In view of the above proof, we get

$$
\nabla \widetilde{\mathcal{L}}_{K} \boldsymbol{\psi}=\boldsymbol{\psi}-\Pi_{K} \boldsymbol{\psi}+\boldsymbol{\psi}_{2}^{*} \quad \forall \boldsymbol{\psi} \in\left[\mathcal{Q}_{1}(K)\right]^{2} .
$$

Using (4.11), we can rewrite (4.2) into

$$
\nabla \mathcal{L}_{K} \boldsymbol{\psi}=\boldsymbol{\psi}-\widetilde{\Pi}_{K} \boldsymbol{\psi}+\boldsymbol{\psi}_{1}^{*} \quad \forall \boldsymbol{\psi} \in\left[\mathcal{Q}_{1}(K)\right]^{2} .
$$

If $\left[\mathcal{Q}_{1}(K)\right]^{2}$ is enriched with the bubble function $[\mathcal{B}(K)]^{2}$, we still have a similar decomposition as well as the estimates. For any $\boldsymbol{\psi} \in\left[\mathcal{Q}_{1}(K) \oplus \mathcal{B}(K)\right]^{2}$, we have the natural decomposition $\boldsymbol{\psi}=\boldsymbol{\psi}_{l}+\boldsymbol{\psi}_{b}$ with $\boldsymbol{\psi}_{l} \in\left[\mathcal{Q}_{1}(K)\right]^{2}$ and $\boldsymbol{\psi}_{b} \in[\mathcal{B}(K)]^{2}$; then a direct calculation gives

$$
\left|\boldsymbol{\psi}_{l}\right|_{1, K} \leq C|\boldsymbol{\psi}|_{1, K} \quad \text { and } \quad\left|\boldsymbol{\psi}_{l}\right|_{2, K} \leq C|\boldsymbol{\psi}|_{2, K} .
$$


Corollary 4.3. For any $\boldsymbol{\psi} \in\left[\mathcal{Q}_{1}(K) \oplus \mathcal{B}(K)\right]^{2}$, we have

$$
\nabla \mathcal{L}_{K} \boldsymbol{\psi}=\boldsymbol{\psi}-\widetilde{\Pi}_{K} \boldsymbol{\psi}+\boldsymbol{\psi}^{*}
$$

Moreover, the residue $\boldsymbol{\psi}^{*}$ admits the estimates

$$
\begin{aligned}
\left|\left(\boldsymbol{s}, \boldsymbol{\psi}^{*}\right)_{K}\right| & \leq C h_{K}^{2}\left(\|\boldsymbol{s}\|_{0, K}|\boldsymbol{\psi}|_{2, K}+\|\operatorname{div} \boldsymbol{s}\|_{0, K}|\boldsymbol{\psi}|_{1, K}\right) \quad \forall \boldsymbol{s} \in \boldsymbol{H}(\operatorname{div}, K), \\
\left\|\boldsymbol{\psi}^{*}\right\|_{0, K} & \leq C\left(d_{K}^{2} / h_{K}\right)|\boldsymbol{\psi}|_{2, K} .
\end{aligned}
$$

Proof. Considering the definition of $\mathcal{L}_{K}$, we see that $\mathcal{L}_{K} \boldsymbol{\psi}_{b}=0$. It follows from (4.13) that $\nabla \mathcal{L}_{K} \boldsymbol{\psi}-\boldsymbol{\psi}=-\widetilde{\Pi}_{K} \boldsymbol{\psi}+\boldsymbol{\psi}^{*}$, which gives (4.15) with $\boldsymbol{\psi}^{*}=\boldsymbol{\psi}_{l}{ }^{*}+\widetilde{\Pi}_{K} \boldsymbol{\psi}_{b}-$ $\psi_{b}$.

It remains to bound the residue. An integration by parts gives

$$
\int_{K} \frac{\partial \boldsymbol{\psi}_{b}}{\partial x} d \boldsymbol{x}=0 \quad \text { and } \quad \int_{K} \frac{\partial \boldsymbol{\psi}_{b}}{\partial y} d \boldsymbol{x}=0
$$

since $\boldsymbol{\psi}_{b}$ vanishes on $\partial K$. Invoking Poincaré's inequality, we have

$$
\left|\boldsymbol{\psi}_{b}\right|_{1, K} \leq C h_{K}\left|\boldsymbol{\psi}_{b}\right|_{2, K} .
$$

By the triangle inequality and (4.14),

$$
\left|\boldsymbol{\psi}_{b}\right|_{2, K} \leq\left|\boldsymbol{\psi}_{l}\right|_{2, K}+|\boldsymbol{\psi}|_{2, K} \leq C|\boldsymbol{\psi}|_{2, K} .
$$

A combination of above two inequalities leads to

$$
\left|\boldsymbol{\psi}_{b}\right|_{1, K} \leq C h_{K}|\boldsymbol{\psi}|_{2, K},
$$

which together with the interpolation estimate (4.21) for $\widetilde{\Pi}_{K}$ gives

$$
\left\|\boldsymbol{\psi}_{b}-\widetilde{\Pi}_{K} \boldsymbol{\psi}_{b}\right\|_{0, K} \leq C h_{K}\left|\boldsymbol{\psi}_{b}\right|_{1, K} \leq C h_{K}^{2}|\boldsymbol{\psi}|_{2, K} .
$$

Combining the above inequality and (4.3) 1 , we get (4.16) 1 .

Note that (4.8) can be improved to

$$
\left\|\boldsymbol{\psi}_{l}^{*}\right\|_{0, K} \leq C\left(d_{K}^{2} / h_{K}\right)\left|\boldsymbol{\psi}_{l}\right|_{2, K} \leq C\left(d_{K}^{2} / h_{K}\right)|\boldsymbol{\psi}|_{2, K},
$$

where we have used (4.14) in the last step. Using the above inequality and (4.18), we obtain

$$
\left\|\boldsymbol{\psi}^{*}\right\|_{0, K} \leq\left\|\boldsymbol{\psi}_{l}^{*}\right\|_{0, K}+\left\|\boldsymbol{\psi}_{b}-\widetilde{\Pi}_{K} \boldsymbol{\psi}_{b}\right\|_{0, K} \leq C\left(d_{K}^{2} / h_{K}\right)|\boldsymbol{\psi}|_{2, K},
$$

which gives $(4.16)_{2}$.

Remark 4.4. In view of Theorem 4.1 and Corollary 4.3, the residue $\boldsymbol{\psi}^{*}$ does not vanish - even the quadrilateral reduces to a parallelogram or a rectangle.

Using (4.13) and (4.15), we have for any $\boldsymbol{\psi}$ in $\boldsymbol{V}_{h}$ or $\boldsymbol{V}_{h}^{*}$,

$$
\nabla \mathcal{L}_{K} \boldsymbol{\psi}=\boldsymbol{\psi}-\widetilde{\Pi}_{K} \boldsymbol{\psi}+\boldsymbol{\psi}^{*}
$$

Denote $\mathcal{T}_{K} \boldsymbol{\psi}:=\boldsymbol{\psi}^{*}$ for any $\boldsymbol{\psi}$ in $\boldsymbol{V}_{h}$ or $\boldsymbol{V}_{h}^{*} . \mathcal{T}_{K}$ is a well-defined linear operator due to the above identity (4.20). Define a global operator $\mathcal{T}_{h}$ by $\left.\mathcal{T}_{h}\right|_{K}:=\mathcal{T}_{K}$. By the locally defined operator $\mathcal{L}_{K}$ we embed it into a global operator $\mathcal{L}_{h}$ with $\left.\mathcal{L}_{h}\right|_{K}=\mathcal{L}_{K}$. Similarly, using (4.11), we may define a global operator $\widetilde{\mathcal{L}}_{h}$ by $\left.\widetilde{\mathcal{L}}_{h}\right|_{K}=\widetilde{\mathcal{L}}_{K}$. 
4.2. Properties for the kinematically linked interpolation operator. Before proceeding, we cite some interpolation estimates for $\boldsymbol{R}_{h}$.

Lemma 4.5. For any $\boldsymbol{u} \in \boldsymbol{H}^{1}(K)$, there exists a constant $C$ such that

$$
\left\|\boldsymbol{u}-\boldsymbol{R}_{K} \boldsymbol{u}\right\|_{0, K} \leq C h_{K}|\boldsymbol{u}|_{1, K} .
$$

Moreover, if $\boldsymbol{u} \in \boldsymbol{H}^{1}(K)$ and $\operatorname{rot} \boldsymbol{u} \in H^{1}(K)$, then

$$
\left\|\operatorname{rot}\left(\boldsymbol{u}-\widetilde{\Pi}_{K} \boldsymbol{u}\right)\right\|_{0, K} \leq C h_{K}|\operatorname{rot} \boldsymbol{u}|_{1, K} .
$$

If the $(1+\alpha)$-Section Condition holds, then

$$
\left\|\operatorname{rot}\left(\boldsymbol{u}-\Pi_{K} \boldsymbol{u}\right)\right\|_{0, K} \leq C\left(h_{K}|\operatorname{rot} \boldsymbol{u}|_{1, K}+h_{K}^{\alpha}\|\operatorname{rot} \boldsymbol{u}\|_{0, K}\right)
$$

for all $\boldsymbol{u} \in \boldsymbol{H}^{1}(K)$ and $\operatorname{rot} \boldsymbol{u} \in H^{1}(K)$.

The interpolation estimate (4.21) is essentially proved in [19, Lemma 7.1] and 2., Theorem 7].1] We refer to [30, Theorem 3.1] for (4.23) and [2, Theorem 8] for (4.22).

Remark 4.6. The interpolation estimate (4.23) is sharp in the sense that the term $h_{K}^{\alpha}\|\operatorname{rot} \boldsymbol{u}\|_{0, K}$ is indispensable. See [2] for a related counterexample.

We shall state a property for operators $\Pi$ and $\widetilde{\Pi}$ which is crucial for reformulating the method.

Lemma 4.7. For any $\boldsymbol{u} \in \boldsymbol{H}^{s}(K)$ with $s>1$, we have

$$
\begin{aligned}
\Pi_{K} \nabla \boldsymbol{u} & =\nabla \Pi_{1} \boldsymbol{u}, \\
\widetilde{\Pi}_{K} \nabla \boldsymbol{u} & =\nabla \Pi_{1} \boldsymbol{u} .
\end{aligned}
$$

Proof. The first identity (4.24) is well known; we refer to [17, Lemma 2.1] and [26, 32.

Given $s$ for which $\widetilde{\Pi}_{K} s$ is well defined, we have

$$
\widetilde{\Pi}_{K} \boldsymbol{s}=\Pi_{K} \boldsymbol{s}+\left(\int_{\hat{K}} \widehat{\operatorname{rot} \hat{\boldsymbol{s}}} \cdot \hat{x}\right)\left(\begin{array}{c}
0 \\
\frac{3}{8}\left(\hat{x}^{2}-1\right)
\end{array}\right)+\left(\int_{\hat{K}} \widehat{\operatorname{rot}} \hat{\boldsymbol{s}} \cdot \hat{y}\right)\left(\begin{array}{c}
\frac{3}{8}\left(1-\hat{y}^{2}\right) \\
0
\end{array}\right) \text {. }
$$

Let $\boldsymbol{s}=\nabla \boldsymbol{u}$. Using $\widehat{\operatorname{rot}} \hat{\boldsymbol{s}}(\hat{\boldsymbol{x}})=J_{K} \operatorname{rot} \boldsymbol{s}(\boldsymbol{F}(\hat{\boldsymbol{x}}))$, we get $\widetilde{\Pi}_{K} \nabla \boldsymbol{u}=\Pi_{K} \nabla \boldsymbol{u}=\nabla \Pi_{1} \boldsymbol{u}$, which gives (4.25).

The following two lemmas concern the estimates for $\mathcal{T}_{h}, \mathcal{L}_{h}$ and $\widetilde{\mathcal{L}}_{H}$.

Lemma 4.8. For any $\boldsymbol{\psi}$ in $\boldsymbol{V}_{h}$ or $\boldsymbol{V}_{h}^{*}$, we have

$$
\left\|\mathcal{T}_{h} \psi\right\|_{0} \leq C h|\psi|_{1}
$$

If the $(1+\alpha)$-Section Condition holds, then the above estimate can be improved to

$$
\left\|\mathcal{T}_{h} \boldsymbol{\psi}\right\|_{0} \leq C\left(\sum_{K \in \mathcal{C}_{h}} h_{K}^{2+4 \alpha}|\boldsymbol{\psi}|_{2, K}^{2}\right)^{1 / 2}
$$

\footnotetext{
${ }^{1}$ In fact, a proof for the interpolation error of the lowest order $\boldsymbol{H}$ (div) Raviart-Thomas element with respect to the $L^{2}$ norm was given in [19, Lemma 7.1] and [2, Theorem 8].
} 
Proof. If $\boldsymbol{\psi} \in \boldsymbol{V}_{h}$, then using (4.8), we have

$$
\left\|\mathcal{T}_{K} \boldsymbol{\psi}\right\|_{0, K}=\left\|\boldsymbol{\psi}_{1}^{*}\right\|_{0, K} \leq C h_{K}|\boldsymbol{\psi}|_{1, K} .
$$

In the case of $\boldsymbol{\psi} \in \boldsymbol{V}_{h}^{*}$, invoking (4.8) and noting (4.21) for $\boldsymbol{R}_{K}=\widetilde{\Pi}_{K}$, we get

$$
\left\|\mathcal{T}_{K} \boldsymbol{\psi}\right\|_{0, K} \leq C h_{K}\left(\left|\boldsymbol{\psi}_{l}\right|_{1, K}+\left|\boldsymbol{\psi}_{b}\right|_{1, K}\right) \leq C h_{K}|\boldsymbol{\psi}|_{1, K},
$$

where we have used $\left|\boldsymbol{\psi}_{l}\right|_{1, K}+\left|\boldsymbol{\psi}_{b}\right|_{1, K} \leq|\boldsymbol{\psi}|_{1, K}+2\left|\boldsymbol{\psi}_{l}\right|_{1, K} \leq C|\boldsymbol{\psi}|_{1, K}$. Summing over all quadrilaterals $K$, the estimate (4.26) follows from (4.28) and (4.29).

If the $(1+\alpha)$-Section Condition holds, the improved estimate (4.27) follows from (4.19) and (4.16) 2 .

Lemma 4.9. For any $\boldsymbol{\psi}$ in $\boldsymbol{V}_{h}$ or $\boldsymbol{V}_{h}^{*}$, we have $\mathcal{L}_{h} \boldsymbol{\psi} \in H_{0}^{1}(\Omega)$ and

$$
\left\|\mathcal{L}_{h} \boldsymbol{\psi}\right\|_{0} \leq C h\left\|\nabla \mathcal{L}_{h} \boldsymbol{\psi}\right\|_{0} \leq C h^{2}|\boldsymbol{\psi}|_{1} .
$$

Similarly, for any $\boldsymbol{\psi}$ in $\boldsymbol{V}_{h}$ or $\boldsymbol{V}_{h}^{*}$, we have $\widetilde{\mathcal{L}}_{h} \boldsymbol{\psi} \in H_{0}^{1}(\Omega)$ and

$$
\left\|\widetilde{\mathcal{L}}_{h} \boldsymbol{\psi}\right\|_{0} \leq C h\left\|\nabla \widetilde{\mathcal{L}}_{h} \boldsymbol{\psi}\right\|_{0} \leq C h^{2}|\boldsymbol{\psi}|_{1} .
$$

Proof. For any $\boldsymbol{\psi}$ in $\boldsymbol{V}_{h}$ or $\boldsymbol{V}_{h}^{*}, \mathcal{L}_{K} \boldsymbol{\psi}$ is completely determined by $\boldsymbol{\psi} \cdot \boldsymbol{\tau}$, so $\mathcal{L}_{h} \boldsymbol{\psi} \in$ $\mathcal{C}^{0}(\bar{\Omega})$. On each element $K, \mathcal{L}_{K} \boldsymbol{\psi} \in \mathcal{E}(K)$, by [16, Theorem 2.1.1], we conclude that $\mathcal{L}_{h} \boldsymbol{\psi} \in H_{0}^{1}(\Omega)$.

Note that $\mathcal{L}_{K} \boldsymbol{\psi}$ vanishes at four vertices of $K$; we have

$$
\left\|\mathcal{L}_{K} \boldsymbol{\psi}\right\|_{0, K}=\left\|\mathcal{L}_{K} \boldsymbol{\psi}-\Pi_{1} \mathcal{L}_{K} \boldsymbol{\psi}\right\|_{0, K} \leq C h_{K}\left\|\nabla \mathcal{L}_{K} \boldsymbol{\psi}\right\|_{0, K}
$$

From (4.20), (4.28), (4.29) and (4.21), it follows that

$$
\left\|\nabla \mathcal{L}_{K} \boldsymbol{\psi}\right\|_{0, K} \leq\left\|\boldsymbol{\psi}-\widetilde{\Pi}_{K} \boldsymbol{\psi}\right\|_{0, K}+\left\|\mathcal{T}_{K} \boldsymbol{\psi}\right\|_{0, K} \leq C h_{K}|\boldsymbol{\psi}|_{1, K}
$$

Combining the above two inequalities, summing over all quadrilaterals $K$, and noting that $\mathcal{L}_{h} \psi \in H_{0}^{1}(\Omega)$, we obtain (4.30).

Noting that $\widetilde{\mathcal{L}}_{K}=\mathcal{L}_{K}$ along each edge of the element $K$ and proceeding along the same line, we obtain $\widetilde{\mathcal{L}}_{h} \in H_{0}^{1}(\Omega)$ and the estimate (4.31).

As an application of the above lemma, we prove a result which is crucial for the $\mathrm{L}^{2}$ error estimate of lower order quadrilateral elements for the Reissner-Mindlin plate [17].

Lemma 4.10. If $\boldsymbol{\psi} \in \boldsymbol{H}_{0}^{1}(\Omega) \cap \boldsymbol{H}^{2}(\Omega)$ and $\boldsymbol{\zeta} \in \boldsymbol{H}(\operatorname{div}, \Omega)$, then

$$
\begin{aligned}
&\left|\left(\boldsymbol{\zeta}, \boldsymbol{\Pi}_{1} \boldsymbol{\psi}-\Pi \boldsymbol{\Pi}_{1} \boldsymbol{\psi}\right)\right| \leq C h^{2}\|\operatorname{div} \boldsymbol{\zeta}\|_{0}\left|\boldsymbol{\Pi}_{1} \boldsymbol{\psi}\right|_{1} \\
&+C h\|\boldsymbol{\zeta}\|_{0}\left\|\operatorname{rot}\left(\boldsymbol{\Pi}_{1} \boldsymbol{\psi}-\Pi \boldsymbol{\Pi}_{1} \boldsymbol{\psi}\right)\right\|_{0}, \\
&\left|\left(\boldsymbol{\zeta}, \boldsymbol{\Pi}_{1} \boldsymbol{\psi}-\widetilde{\Pi} \boldsymbol{\Pi}_{1} \boldsymbol{\psi}\right)\right| \leq C h^{2}\|\operatorname{div} \boldsymbol{\zeta}\|_{0}\left|\boldsymbol{\Pi}_{1} \boldsymbol{\psi}\right|_{1} .
\end{aligned}
$$

Moreover, if the $(1+\alpha)$-Section Condition holds, then we may bound (4.33) as

$$
\left|\left(\boldsymbol{\zeta}, \boldsymbol{\Pi}_{1} \boldsymbol{\psi}-\Pi \boldsymbol{\Pi}_{1} \boldsymbol{\psi}\right)\right| \leq C h^{2}\|\boldsymbol{\zeta}\|_{\boldsymbol{H}(\mathrm{div})}\|\boldsymbol{\psi}\|_{2}+C h^{1+\alpha}\|\boldsymbol{\zeta}\|_{0}\|\operatorname{rot} \boldsymbol{\psi}\|_{0} .
$$

Proof. Using (4.12), we have

$$
\left(\boldsymbol{\zeta}, \boldsymbol{\Pi}_{1} \psi-\Pi \boldsymbol{\Pi}_{1} \psi\right)=\left(\zeta, \nabla \widetilde{\mathcal{L}}_{h} \boldsymbol{\Pi}_{1} \psi\right)-\sum_{K \in \mathcal{C}_{h}} \int_{K} \boldsymbol{\zeta}\left(\boldsymbol{\Pi}_{1} \psi\right)_{2}^{*} d \boldsymbol{x} .
$$

Noting that $\widetilde{\mathcal{L}}_{h} \boldsymbol{\Pi}_{1} \boldsymbol{\psi} \in \boldsymbol{H}_{0}^{1}(\Omega)$ and $\boldsymbol{\zeta} \in \boldsymbol{H}(\operatorname{div}, \Omega)$, an integration by parts gives

$$
\left(\boldsymbol{\zeta}, \nabla \widetilde{\mathcal{L}}_{h} \Pi_{1} \psi\right)=-\left(\operatorname{div} \boldsymbol{\zeta}, \widetilde{\mathcal{L}}_{h} \Pi_{1} \psi\right) .
$$


It follows from Lemma 4.9 that

$$
\left|\left(\boldsymbol{\zeta}, \nabla \widetilde{\mathcal{L}}_{h} \boldsymbol{\Pi}_{1} \psi\right)\right| \leq C h^{2}\|\operatorname{div} \boldsymbol{\zeta}\|_{0}\left|\boldsymbol{\Pi}_{1} \boldsymbol{\psi}\right|_{1} .
$$

Invoking (4.3) 2 gives

$$
\left|\sum_{K \in \mathcal{C}_{h}} \int_{K} \boldsymbol{\zeta}\left(\boldsymbol{\Pi}_{1} \boldsymbol{\psi}\right)_{2}^{*} d \boldsymbol{x}\right| \leq C h\|\boldsymbol{\zeta}\|_{0}\left\|\operatorname{rot}\left(\boldsymbol{\Pi}_{1} \boldsymbol{\psi}-\Pi \boldsymbol{\Pi}_{1} \boldsymbol{\psi}\right)\right\|_{0}
$$

A combination of above two inequalities gives (4.33). The last estimate (4.35) follows from (4.33) and (4.23).

Proceeding along the same line as the above procedure and using Theorem 4.2 , we obtain (4.34).

Remark 4.11. Estimates similar to (4.33) have been established in 28, Lemma 3.1], 17, Lemma 4.2] and [30, Lemma 3.5], but the estimate (4.34) appears to be new. The proof is different from methodologies in [17, 28, 30, which are essentially based upon the Hemholtz decomposition [14].

4.3. Reformulation of the method. Based on Theorems 4.1 and 4.2 , we introduce a general variational formulation which covers the stabilized MITC4, MIN4 and FMIN4 elements.

Problem 4.12. Find $\left(\phi_{h}, \omega_{h}\right) \in \boldsymbol{V}_{h} \times W_{h}$ such that

$$
\mathcal{B}_{h}\left(\phi_{h}, \omega_{h} ; \boldsymbol{\psi}, v\right)=\ell_{h}(\boldsymbol{\psi}, v) \quad \forall(\boldsymbol{\psi}, v) \in \boldsymbol{V}_{h} \times W_{h},
$$

where

$$
\begin{aligned}
\mathcal{B}_{h}\left(\phi_{h}, \omega_{h} ; \boldsymbol{\psi}, v\right) & :=a\left(\boldsymbol{\phi}_{h}, \boldsymbol{\psi}\right) \\
& +\sum_{K \in \mathcal{C}_{h}} \frac{\lambda}{t^{2}+\alpha_{K} h_{K}^{2}}\left(\boldsymbol{R}_{K}\left(\nabla \omega_{h}-\boldsymbol{\phi}_{h}\right)+\mathcal{T}_{K} \boldsymbol{\phi}_{h}, \boldsymbol{R}_{K}(\nabla v-\boldsymbol{\psi})+\mathcal{T}_{K} \boldsymbol{\psi}\right) .
\end{aligned}
$$

$\gamma_{h}$ is defined locally as $\gamma_{\left.h\right|_{K}}=\left[\lambda /\left(t^{2}+\alpha_{K} h_{K}^{2}\right)\right]\left(\boldsymbol{R}_{K}\left(\nabla \omega_{h}-\boldsymbol{\phi}_{h}\right)+\mathcal{T}_{K} \boldsymbol{\phi}_{h}\right)$.

- The stabilized MITC4 element: $\mathcal{T}_{K}=\mathbf{0}, \boldsymbol{R}_{K}=\Pi_{K}$, and $\ell_{h}(\boldsymbol{\psi}, v)=(g, v)$.

- The FMIN4 element: $\mathcal{T}_{K}=\mathbf{0}, \boldsymbol{R}_{K}=\widetilde{\Pi}_{K}$, and $\ell_{h}(\boldsymbol{\psi}, v)=\left(g, v+\widetilde{\mathcal{L}}_{h} \boldsymbol{\psi}\right)$.

- The MIN4 element: $\boldsymbol{R}_{K}=\widetilde{\Pi}_{K}$ and $\ell_{h}(\boldsymbol{\psi}, v)=\left(g, v+\mathcal{L}_{h} \boldsymbol{\psi}\right)$.

Remark 4.13. If we let the right-hand side of the MIN4 and FMIN4 elements be the same as the stabilized MITC4 element, then in view of Lemma 4.9 and as that in [29, §4], we can show that the difference between the modified MIN4, FMIN4 elements and the original ones is $\mathcal{O}\left(h^{2}\right)$ with respect to the $\||\cdot| \mid-$-norm defined below.

If the quadrilateral changes to a rectangle, then we can use the standard static condensation procedure 33] to reformulate the Q4BL element as follows.

Find $\left(\phi_{h}, \omega_{h}\right) \in \boldsymbol{V}_{h} \times W_{h}$ such that $\mathcal{B}_{h}\left(\phi_{h}, \omega_{h} ; \boldsymbol{\psi}, v\right)=\ell_{h}(\boldsymbol{\psi}, v)$ for all $(\boldsymbol{\psi}, v) \in$ $\boldsymbol{V}_{h} \times W_{h}$. Here $\mathcal{B}_{h}$ and $\ell_{h}$ are defined respectively as

$$
\begin{aligned}
\mathcal{B}_{h}(\boldsymbol{\eta}, w ; \boldsymbol{\psi}, v):=a(\boldsymbol{\eta}, \boldsymbol{\psi})-\sum_{K \in \mathcal{C}_{h}}\left(\left(B_{K}^{-1}-\lambda^{-1} t^{2} \boldsymbol{I}\right) A \boldsymbol{\eta}, A \boldsymbol{\psi}\right) \\
+\sum_{K \in \mathcal{C}_{h}}\left(B_{K} \boldsymbol{\Pi}_{0}\left(\widetilde{\Pi}_{K}(\nabla w-\boldsymbol{\eta})+\mathcal{T}_{K} \boldsymbol{\eta}\right)-\left(B_{K}^{-1}-\lambda^{-1} t^{2} \boldsymbol{I}\right) A \boldsymbol{\eta},\right. \\
\left.\boldsymbol{\Pi}_{0}\left(\widetilde{\Pi}_{K}(\nabla v-\boldsymbol{\psi})+\mathcal{\mathcal { T }}_{K} \boldsymbol{\psi}\right)-\left(B_{K}^{-1}-\lambda^{-1} t^{2} \boldsymbol{I}\right) A \boldsymbol{\psi}\right)
\end{aligned}
$$


with $B_{K}:=\left(\lambda^{-1} t^{2}+\frac{16}{81}|K| A_{K}^{-1}\right)^{-1}$, where

$$
A_{K}=\left(\begin{array}{ll}
a\left(\phi_{1}, \phi_{1}\right) & a\left(\phi_{1}, \phi_{2}\right) \\
a\left(\phi_{1}, \phi_{2}\right) & a\left(\phi_{2}, \phi_{2}\right)
\end{array}\right),
$$

and $|K|$ is the area of element $K, \phi$ is the quadratic bubble function in $\mathcal{B}(K)$, and $\boldsymbol{\phi}_{1}=(\phi, 0), \boldsymbol{\phi}_{2}=(0, \phi)$. The differential operator $A$ is defined as $(A \boldsymbol{\eta}, \boldsymbol{\psi})=$ $-a(\boldsymbol{\eta}, \boldsymbol{\psi})$. It is easy to see that

$$
\left\|B_{K}\right\|=\mathcal{O}\left(\frac{1}{t^{2}+h_{K}^{2}}\right) \quad \text { and } \quad\left\|B_{K}^{-1}-\lambda^{-1} t^{2} \boldsymbol{I}\right\|=\mathcal{O}\left(h_{K}^{2}\right),
$$

where $\|\cdot\|$ denotes the matrix norm by $\|A\|:=\sup _{x \in \mathbb{R}^{2},|x|=1}|A x|$. The linear form $\ell_{h}$ is defined as $\ell_{h}(v, \boldsymbol{\psi}):=\left(g, v+\mathcal{L}_{h} \boldsymbol{\psi}\right)$.

Consequently, the Q4BL element is actually the stabilized MITC4 element introduced in [25] or [26, Method 4.1 with $k=1$ ] provided that the operator $\boldsymbol{\Pi}_{0}$ is replaced by $\boldsymbol{I}$ and $\mathcal{T}_{K}$ vanishes.

It is reported in [39] that there is one zero energy mode for the Q4BL element, which is clear from (4.38). Such defect is due to the lack of stability. To overcome this difficulty, Auricchio and Lovadina 7 proposed an augmented variational formulation and analyzed rectangular elements. This modified Q4BL element behaves quite well in the benchmark computation, whereas the analysis therein indicates that it is locking since the right-hand side of the error bound depends on $\|\gamma\|_{1}$ and $\|\omega\|_{3}$, which is of $\mathcal{O}\left(t^{-1 / 2}\right)$ for the clamped plate, and even worse for other boundary conditions [6]. In $\S 6$, we will modify their variational formulation as that in [15] and give a complete analysis for general quadrilateral elements.

\section{ERror estimate}

FOR THE STABILIZED MITC4, MIN4, AND FMIN4 ELEMENTS

To analyze Problem 4.12, we introduce some mesh-dependent norms. For any $\boldsymbol{\psi} \in \boldsymbol{V}_{h}$ or $\boldsymbol{V}_{h}^{*}, v \in W_{h}$ and $\boldsymbol{s} \in \boldsymbol{\Gamma}_{h}$ or $\boldsymbol{M}_{h}$, define

$$
\|\boldsymbol{\psi}, v\|:=\|\boldsymbol{\psi}\|_{1}+\|v\|_{1}+|\boldsymbol{\psi}, v|_{h}
$$

and

$$
\|\boldsymbol{s}\|_{h}:=\left(\sum_{K \in \mathcal{C}_{h}}\left(t^{2}+\alpha_{K} h_{K}^{2}\right)\|\boldsymbol{s}\|_{0, K}^{2}\right)^{1 / 2}, \quad\|\boldsymbol{\psi}, v, \boldsymbol{s}\|:=\|\boldsymbol{\psi}, v\|+\|\boldsymbol{s}\|_{h},
$$

where $|\boldsymbol{\psi}, v|_{h}$ is defined as

$$
|\boldsymbol{\psi}, v|_{h}:=\left(\sum_{K \in \mathcal{C}_{h}}\left(t^{2}+\alpha_{K} h_{K}^{2}\right)^{-1}\left\|\boldsymbol{R}_{K}(\nabla v-\boldsymbol{\psi})\right\|_{0, K}^{2}\right)^{1 / 2} .
$$

Remark 5.1. If $\boldsymbol{R}_{K}=\Pi_{K}$, it follows from (4.28) that there exist constants $C_{1}$ and $C_{2}$ such that

$$
C_{1}|\boldsymbol{\psi}, v|_{h} \leq\left(\sum_{K \in \mathcal{C}_{h}}\left(t^{2}+\alpha_{K} h_{K}^{2}\right)^{-1}\left\|\nabla\left(v+\mathcal{L}_{K} \boldsymbol{\psi}\right)-\boldsymbol{\psi}\right\|_{0, K}^{2}\right)^{1 / 2}+|\boldsymbol{\psi}|_{1}
$$

and

$$
\left(\sum_{K \in \mathcal{C}_{h}}\left(t^{2}+\alpha_{K} h_{K}^{2}\right)^{-1}\left\|\nabla\left(v+\mathcal{L}_{K} \boldsymbol{\psi}\right)-\boldsymbol{\psi}\right\|_{0, K}^{2}\right)^{1 / 2} \leq C_{2}\left(|\boldsymbol{\psi}, v|_{h}+|\boldsymbol{\psi}|_{1}\right)
$$


If $\boldsymbol{R}_{K}=\widetilde{\Pi}_{K}$, using (4.11) we obtain

$$
|\boldsymbol{\psi}, v|_{h}=\left(\sum_{K \in \mathcal{C}_{h}}\left(t^{2}+\alpha_{K} h_{K}^{2}\right)^{-1}\left\|\nabla\left(v+\widetilde{\mathcal{L}}_{K} \boldsymbol{\psi}\right)-\boldsymbol{\psi}\right\|_{0, K}^{2}\right)^{1 / 2}
$$

Define a piecewise constant $\mu$ by

$$
\mu_{\left.\right|_{K}}:=\mu_{K}:=t^{2} /\left(t^{2}+\alpha_{K} h_{K}^{2}\right)
$$

The following lemma indicates that the above mesh-dependent norms admit optimal approximation error bounds.

Lemma 5.2. If $(\phi, \omega, \gamma)$ are solutions of Problem 2.1, then

$$
\begin{aligned}
& \inf _{\boldsymbol{\psi} \in \boldsymbol{V}_{h} \text { or } \boldsymbol{V}_{h}^{*}, v \in W_{h}}\|\boldsymbol{\phi}-\boldsymbol{\psi}, \omega-v\| \leq C h\left(\|\boldsymbol{\phi}\|_{2}+\|\omega\|_{2}\right), \\
& \inf _{\boldsymbol{s} \in \boldsymbol{M}_{h}}\|\boldsymbol{\gamma}-\boldsymbol{s}\|_{h} \leq C h\left(\|\boldsymbol{\gamma}\|_{0}+t\|\boldsymbol{\gamma}\|_{1}\right), \\
& \inf _{\boldsymbol{s} \in \boldsymbol{\Gamma}_{h}}\|\boldsymbol{\gamma}-\boldsymbol{s}\|_{h} \leq C h\left(\|\boldsymbol{\gamma}\|_{0}+t\|\boldsymbol{\gamma}\|_{1}+\|\boldsymbol{\phi}\|_{2}\right) .
\end{aligned}
$$

Proof. Let $\boldsymbol{\psi}=\Pi_{1} \phi$ and $v=\Pi_{1} \omega_{1}$. Using Lemma 4.7, we obtain $\boldsymbol{R}_{K} \nabla \Pi_{1} \omega=$ $\boldsymbol{R}_{K} \nabla \omega$. Therefore,

$$
\boldsymbol{R}_{K}\left(\nabla\left(\omega-\Pi_{1} \omega\right)-\left(\phi-\Pi_{1} \phi\right)\right)=\boldsymbol{R}_{K}\left(\Pi_{1} \phi-\phi\right),
$$

which implies $\left|\phi-\Pi_{1} \phi, \omega-\Pi_{1} \omega\right|_{h}=\left|\phi-\Pi_{1} \phi, 0\right|_{h}$. The standard interpolation error estimate for $\boldsymbol{\Pi}_{1}$ and $\boldsymbol{R}_{h}$ yields

$$
\begin{aligned}
\left\|\boldsymbol{R}_{K}\left(\boldsymbol{\phi}-\boldsymbol{\Pi}_{1} \phi\right)\right\|_{0, K} & \leq\left\|\left(\boldsymbol{R}_{K}-\boldsymbol{I}\right)\left(\boldsymbol{\phi}-\boldsymbol{\Pi}_{1} \phi\right)\right\|_{0, K}+\left\|\boldsymbol{\phi}-\boldsymbol{\Pi}_{1} \boldsymbol{\phi}\right\|_{0, K} \\
& \leq C h_{K}^{2}\|\boldsymbol{\phi}\|_{2, K} .
\end{aligned}
$$

Therefore,

$$
\left|\phi-\Pi_{1} \phi, \omega-\Pi_{1} \omega\right|_{h} \leq C h\|\phi\|_{2} .
$$

This inequality together with the standard interpolation result for the bilinear element gives (5.3).

Putting $s=\Pi_{0} \gamma \in M_{h}$ in the left-hand side of (5.4), we have

$$
\left\|\boldsymbol{\gamma}-\boldsymbol{\Pi}_{0} \boldsymbol{\gamma}\right\|_{h} \leq t\left\|\boldsymbol{\gamma}-\boldsymbol{\Pi}_{0} \boldsymbol{\gamma}\right\|_{0}+C h\left\|\boldsymbol{\gamma}-\boldsymbol{\Pi}_{0} \boldsymbol{\gamma}\right\|_{0} \leq C h\left(t\|\boldsymbol{\gamma}\|_{1}+\|\gamma\|_{0}\right),
$$

which leads to (5.4).

Define $\bar{\gamma}:=\lambda \mu t^{-2}\left(\nabla \Pi_{1} \omega-\boldsymbol{R}_{h} \boldsymbol{\Pi}_{1} \phi\right)$. By Lemma 4.7 we conclude that $\bar{\gamma} \in \boldsymbol{\Gamma}_{h}$, and we may write $\bar{\gamma}$ as

$$
\bar{\gamma}=\lambda \mu t^{-2}\left(\boldsymbol{R}_{h}(\nabla \omega-\phi)+\boldsymbol{R}_{h}\left(\boldsymbol{\phi}-\boldsymbol{\Pi}_{1} \phi\right)\right)=\mu \boldsymbol{R}_{h} \gamma+\lambda \mu t^{-2} \boldsymbol{R}_{h}\left(\boldsymbol{\phi}-\boldsymbol{\Pi}_{1} \phi\right) .
$$

Therefore, $\boldsymbol{\gamma}-\overline{\boldsymbol{\gamma}}=(1-\mu) \boldsymbol{\gamma}+\mu\left(\boldsymbol{\gamma}-\boldsymbol{R}_{h} \boldsymbol{\gamma}\right)+\lambda \mu t^{-2} \boldsymbol{R}_{h}\left(\boldsymbol{\Pi}_{1} \boldsymbol{\phi}-\boldsymbol{\phi}\right)$. It follows that

$$
\begin{aligned}
\|\gamma-\bar{\gamma}\|_{h} & \leq\left(\sum_{K \in \mathcal{C}_{h}} t^{2}\left(1-\mu_{K}\right)^{2} / \mu_{K}\|\gamma\|_{0, K}^{2}\right)^{1 / 2}+\left(\sum_{K \in \mathcal{C}_{h}} t^{2} \mu_{K}\left\|\boldsymbol{\gamma}-\boldsymbol{R}_{K} \gamma\right\|_{0, K}^{2}\right)^{1 / 2} \\
& +\left(\sum_{K \in \mathcal{C}_{h}} \lambda^{2} \mu_{K} t^{-2}\left\|\boldsymbol{R}_{K}\left(\boldsymbol{\phi}-\mathbf{\Pi}_{1} \phi\right)\right\|_{0, K}^{2}\right)^{1 / 2} \\
& \leq C h\left(\|\gamma\|_{0}+t\|\gamma\|_{1}+\|\boldsymbol{\phi}\|_{2}\right),
\end{aligned}
$$

where we have used

$$
t^{2}\left(1-\mu_{K}\right)^{2} / \mu_{K} \leq t^{2}\left(1-\mu_{K}\right) / \mu_{K}=\alpha_{K} h_{K}^{2}
$$

and (5.7) to bound the first and the last term in the right-hand side of the above inequality, respectively. 
A direct consequence of (5.5) is

Corollary 5.3. Let $(\phi, \omega, \gamma)$ be solutions of Problem 2.1, If we define $\widehat{\gamma}:=\mu \lambda t^{-2}\left(\nabla\left(\Pi_{1} \omega+\mathcal{L}_{h} \Pi_{1} \phi\right)-\Pi_{1} \phi\right) \quad$ and $\quad \widetilde{\gamma}:=\mu \lambda t^{-2}\left(\nabla\left(\Pi_{1} \omega+\widetilde{\mathcal{L}}_{h} \Pi_{1} \phi\right)-\Pi_{1} \phi\right)$, then

$$
\begin{aligned}
& \|\boldsymbol{\gamma}-\widehat{\gamma}\|_{h} \leq C h\left(\|\boldsymbol{\gamma}\|_{0}+t\|\boldsymbol{\gamma}\|_{1}+\|\boldsymbol{\phi}\|_{2}\right)+C h^{2 \alpha}\|\boldsymbol{\phi}\|_{2}, \\
& \|\boldsymbol{\gamma}-\widetilde{\boldsymbol{\gamma}}\|_{h} \leq C h\left(\|\boldsymbol{\gamma}\|_{0}+t\|\boldsymbol{\gamma}\|_{1}+\|\boldsymbol{\phi}\|_{2}\right) .
\end{aligned}
$$

Proof. By Lemma 4.7, $\widehat{\gamma}=\bar{\gamma}+\lambda \mu t^{-2} \mathcal{T}_{h} \boldsymbol{\Pi}_{1} \phi$ with $\boldsymbol{R}_{h}=\Pi$. Therefore,

$$
\|\gamma-\widehat{\gamma}\|_{h} \leq\|\gamma-\bar{\gamma}\|_{h}+\left\|\lambda \mu t^{-2} \mathcal{T}_{h} \Pi_{1} \phi\right\|_{h}
$$

Using (4.27) 1 , we obtain

$$
\left\|\lambda \mu t^{-2} \mathcal{T}_{h} \Pi_{1} \phi\right\|_{h} \leq C\left(\sum_{K \in \mathcal{C}_{h}} \mu_{K} t^{-2}\left(h_{K}^{4}+h_{K}^{2+4 \alpha}\right)\left\|\boldsymbol{\Pi}_{1} \phi\right\|_{2, K}^{2}\right)^{1 / 2} \leq C\left(h+h^{2 \alpha}\right)\|\phi\|_{2},
$$

thus $(5.9)_{1}$ follows.

Note that $\widetilde{\gamma}=\bar{\gamma}$ with $\boldsymbol{R}_{h}=\widetilde{\Pi}$, invoking (5.5) leads to (5.9) ${ }_{2}$.

We have the coercivity inequality for Problem 4.12 as follows.

Lemma 5.4. There exists a constant $C$ such that

$$
\mathcal{B}_{h}(\boldsymbol{\psi}, v ; \boldsymbol{\psi}, v) \geq C\|\boldsymbol{\psi}, v\|^{2} \quad \forall(\boldsymbol{\psi}, v) \in \boldsymbol{V}_{h} \times W_{h} .
$$

The proof of (5.10) for the stabilized MITC4 element is referred to 26, Theorem 3.5]. Proceeding along the same line as [26, Theorem 3.5] and using Remark 5.1, we may establish (5.10) for the MIN4 and FMIN4 elements. A combination of the above interpolation and stability results gives

Theorem 5.5. Let $(\phi, \omega, \gamma)$ and $\left(\phi_{h}, \omega_{h}, \gamma_{h}\right)$ be solutions of Problems 2.1 and 4.12 , respectively. If the $(1+\alpha)$-Section Condition holds, then for the MIN4 element,

$$
\left\|\boldsymbol{\phi}-\boldsymbol{\phi}_{h}, \omega-\omega_{h}, \gamma-\gamma_{h}\right\| \leq C h\left(\|g\|_{-1}+t\|g\|_{0}\right)+C h^{2 \alpha}\|g\|_{-1} .
$$

For the stabilized MITC4 and FMIN4 elements,

$$
\left\|\phi-\phi_{h}, \omega-\omega_{h}, \gamma-\gamma_{h}\right\| \leq C h\left(\|g\|_{-1}+t\|g\|_{0}\right) .
$$

Proof. We start from the MIN4 element. Define $\boldsymbol{\psi}:=\phi_{h}-\Pi_{1} \phi$ and $v:=\omega_{h}-\Pi_{1} \omega$. We expand $\mathcal{B}_{h}(\boldsymbol{\psi}, v ; \boldsymbol{\psi}, v)$ as

$$
\begin{aligned}
\mathcal{B}_{h}\left(\phi_{h}-\Pi_{1} \boldsymbol{\phi}, \omega_{h}-\Pi_{1} \omega ; \boldsymbol{\psi}, v\right) & =\mathcal{B}_{h}\left(\boldsymbol{\phi}_{h}, \omega_{h} ; \boldsymbol{\psi}, v\right)-\mathcal{B}_{h}\left(\boldsymbol{\Pi}_{1} \boldsymbol{\phi}, \Pi_{1} \omega ; \boldsymbol{\psi}, v\right) \\
& =\left(g, v+\mathcal{L}_{h} \boldsymbol{\psi}\right)-\mathcal{B}_{h}\left(\boldsymbol{\Pi}_{1} \boldsymbol{\phi}, \Pi_{1} \omega ; \boldsymbol{\psi}, v\right) \\
& =\mathcal{B}\left(\boldsymbol{\phi}, \omega ; \boldsymbol{\psi}, v+\mathcal{L}_{h} \boldsymbol{\psi}\right)-\mathcal{B}_{h}\left(\boldsymbol{\Pi}_{1} \boldsymbol{\phi}, \Pi_{1} \omega ; \boldsymbol{\psi}, v\right) \\
& =a\left(\boldsymbol{\phi}-\boldsymbol{\Pi}_{1} \boldsymbol{\phi}, \boldsymbol{\psi}\right)+\left(\boldsymbol{\gamma}-\widehat{\boldsymbol{\gamma}}, \nabla\left(v+\mathcal{L}_{h} \boldsymbol{\psi}\right)-\boldsymbol{\psi}\right) .
\end{aligned}
$$

It follows that

$$
\left|\mathcal{B}_{h}\left(\phi_{h}-\Pi_{1} \phi, \omega_{h}-\Pi_{1} \omega ; \boldsymbol{\psi}, v\right)\right| \leq C\left(\left\|\boldsymbol{\phi}-\Pi_{1} \phi\right\|_{1}+\|\boldsymbol{\gamma}-\widehat{\gamma}\|_{h}\right)\|\boldsymbol{\psi}, v\|,
$$

which together with the stability property (5.10) leads to

$$
\left\|\phi_{h}-\Pi_{1} \phi, \omega_{h}-\Pi_{1} \omega\right\| \leq C h\left(\|g\|_{-1}+t\|g\|_{0}\right)+C h^{2 \alpha}\|g\|_{-1} .
$$

By definition, $\left\|\widehat{\gamma}-\gamma_{h}\right\|_{h} \leq \sqrt{\lambda}\left|\phi_{h}-\Pi_{1} \phi, \omega_{h}-\Pi_{1} \omega\right|_{h}$. It easily follows that

$$
\left\|\phi_{h}-\Pi_{1} \phi, \omega_{h}-\Pi_{1} \omega, \widehat{\gamma}-\gamma_{h}\right\| \leq C h\left(\|g\|_{-1}+t\|g\|_{0}\right)+C h^{2 \alpha}\|g\|_{-1} .
$$


The final estimate (5.11) now follows directly from the triangle inequality, the interpolation estimate (5.3), and (5.9) $)_{1}$.

The proof for the FMIN4 element and the stabilized MITC4 element is the same. As to the FMIN4 element, we have

$$
\mathcal{B}_{h}\left(\phi_{h}-\Pi_{1} \phi, \omega_{h}-\Pi_{1} \omega ; \boldsymbol{\psi}, v\right)=a\left(\boldsymbol{\phi}-\Pi_{1} \phi, \boldsymbol{\psi}\right)+\left(\boldsymbol{\gamma}-\widetilde{\boldsymbol{\gamma}}, \nabla\left(v+\widetilde{\mathcal{L}}_{h} \boldsymbol{\psi}\right)-\boldsymbol{\psi}\right) .
$$

This gives $\left\|\phi_{h}-\Pi_{1} \phi, \omega_{h}-\Pi_{1} \omega\right\| \leq C h\left(\|g\|_{-1}+t\|g\|_{0}\right)$ by (5.9) 2 and (5.10).

As to the stabilized MITC4 element,

$$
\begin{aligned}
\mathcal{B}_{h}\left(\phi_{h}-\Pi_{1} \phi, \omega_{h}-\Pi_{1} \omega ; \boldsymbol{\psi}, v\right)= & a\left(\boldsymbol{\phi}-\Pi_{1} \boldsymbol{\phi}, \boldsymbol{\psi}\right) \\
& +(\boldsymbol{\gamma}, \Pi \boldsymbol{\psi}-\boldsymbol{\psi})+(\boldsymbol{\gamma}-\bar{\gamma}, \Pi(\nabla v-\boldsymbol{\psi})) .
\end{aligned}
$$

It follows from Lemma 4.5, (5.5), and the stability property (5.10) that

$$
\left\|\phi_{h}-\Pi_{1} \phi, \omega_{h}-\Pi_{1} \omega\right\| \leq C h\left(\|g\|_{-1}+t\|g\|_{0}\right) .
$$

The remaining part of the proof may be proceeded as that in the MIN4 element.

In what follows, we turn to the $\mathrm{L}^{2}$ estimate. Define an auxiliary problem as

Problem 5.6. Find $(\boldsymbol{\psi}, z) \in \boldsymbol{V} \times W$ such that

$$
\mathcal{B}(\boldsymbol{m}, n ; \boldsymbol{\psi}, z)=\left(\boldsymbol{\phi}-\boldsymbol{\phi}_{h}, \boldsymbol{m}\right)+\left(\omega-\omega_{h}, n\right) \quad \forall(\boldsymbol{m}, n) \in \boldsymbol{V} \times W .
$$

Define $s:=\lambda t^{-2}(\nabla z-\boldsymbol{\psi})$.

The regularity property for the solution of the above problem follows from (2.5), (2.6), Lemma 2.2, and Remark 2.3 as

$$
\|\boldsymbol{\psi}\|_{2}+\|z\|_{3}+\|\boldsymbol{s}\|_{\boldsymbol{H}(\mathrm{div})}+t\|\boldsymbol{s}\|_{1}+t^{-1}\|\operatorname{rot} \boldsymbol{\psi}\|_{0} \leq C\left(\left\|\boldsymbol{\phi}-\boldsymbol{\phi}_{h}\right\|_{0}+\left\|\omega-\omega_{h}\right\|_{0}\right) .
$$

For the MIN4 element, we denote $\underline{\boldsymbol{\psi}}=\Pi_{1} \boldsymbol{\psi}, \underline{z}=\Pi_{1} z+\mathcal{L}_{h} \underline{\boldsymbol{\psi}}$, and $\hat{\boldsymbol{s}}=$ $\lambda \mu t^{-2}(\nabla \underline{z}-\underline{\psi})$, while for the FMIN4 element, the definitions for $\underline{\underline{\psi}}, \underline{z}$ and $\hat{\boldsymbol{s}}$ are the same except that $\mathcal{L}_{h}$ is replaced by $\widetilde{\mathcal{L}}_{h}$. As to the stabilized MITC4 element, we define $\underline{\psi}=\Pi_{1} \psi, \underline{z}=\Pi_{1} z$, and $\hat{s}=\lambda \mu t^{-2}(\nabla \underline{z}-\Pi \underline{\psi})$. It follows from (5.2) that

$$
\|\mu \boldsymbol{s}-\hat{\boldsymbol{s}}\|_{h} \leq\|(1-\mu) \boldsymbol{s}\|_{h}+\|\boldsymbol{s}-\hat{\boldsymbol{s}}\|_{h} \leq C h\|\boldsymbol{s}\|_{0}+\|\boldsymbol{s}-\hat{\boldsymbol{s}}\|_{h} .
$$

In view of Corollary [5.3, we get

$$
\|\mu \boldsymbol{s}-\hat{\boldsymbol{s}}\|_{h} \leq \begin{cases}C h\left(\|\boldsymbol{\psi}\|_{2}+\|\boldsymbol{s}\|_{0}+t\|\boldsymbol{s}\|_{1}\right)+C h^{2 \alpha}\|\boldsymbol{\psi}\|_{2}, & \text { MIN4, } \\ C h\left(\|\boldsymbol{\psi}\|_{2}+\|\boldsymbol{s}\|_{0}+t\|\boldsymbol{s}\|_{1}\right), & \text { FMIN4 }\end{cases}
$$

Using (5.8) with $\boldsymbol{R}_{h}=\Pi$, we obtain

$$
\|\mu \boldsymbol{s}-\hat{\boldsymbol{s}}\|_{h} \leq C h\left(\|\boldsymbol{\psi}\|_{2}+\|\boldsymbol{s}\|_{0}+t\|\boldsymbol{s}\|_{1}\right), \quad \text { STABILIZED MITC4. }
$$

With this auxiliary problem (5.6) and exploiting the dual argument, we obtain the $\mathrm{L}^{2}$ estimate as

Theorem 5.7. Let $(\phi, \omega, \gamma)$ and $\left(\phi_{h}, \omega_{h}, \gamma_{h}\right)$ be solutions of Problems 2.1 and 4.12 , respectively. If the $(1+\alpha)$-Section Condition holds, then for the MIN 4 element,

$$
\left\|\phi-\phi_{h}\right\|_{0}+\left\|\omega-\omega_{h}\right\|_{0} \leq C\left(h^{2}+h^{1+2 \alpha}\right)\left(\|g\|_{-1}+t\|g\|_{0}\right)+C h^{4 \alpha}\|g\|_{-1},
$$

and for the stabilized MITC4 element,

$$
\left\|\phi-\phi_{h}\right\|_{0}+\left\|\omega-\omega_{h}\right\|_{0} \leq C h^{1+\alpha} t\|g\|_{-1}+C h^{2}\|g\|_{0} .
$$


For the FMIN4 element,

$$
\left\|\phi-\phi_{h}\right\|_{0}+\left\|\omega-\omega_{h}\right\|_{0} \leq C h^{2}\left(\|g\|_{-1}+t\|g\|_{0}\right) .
$$

Proof. Inserting $\boldsymbol{m}=\boldsymbol{\phi}-\boldsymbol{\phi}_{h}$ and $n=\omega-\omega_{h}$ into (5.14), we get

$$
\begin{aligned}
\left\|\boldsymbol{\phi}-\boldsymbol{\phi}_{h}\right\|_{0}^{2}+\left\|\omega-\omega_{h}\right\|_{0}^{2} & =\mathcal{B}\left(\boldsymbol{\phi}-\boldsymbol{\phi}_{h}, \omega-\omega_{h} ; \boldsymbol{\psi}, z\right)=(g, z)-\mathcal{B}\left(\boldsymbol{\phi}_{h}, \omega_{h} ; \boldsymbol{\psi}, z\right) \\
& =(g, z-\underline{z})+\left[\mathcal{B}_{h}\left(\boldsymbol{\phi}_{h}, \omega_{h} ; \underline{\boldsymbol{\psi}}, \Pi_{1} z\right)-\mathcal{B}\left(\boldsymbol{\phi}_{h}, \omega_{h} ; \boldsymbol{\psi}, z\right)\right] .
\end{aligned}
$$

Noting that $-\operatorname{div} \gamma=g$ and $z, \underline{z} \in H_{0}^{1}(\Omega)$, an integration by parts gives

$$
(g, z-\underline{z})=(\gamma, \nabla z-\nabla \underline{z}) .
$$

We write the second term on the right-hand side of (5.22) as

$\mathcal{B}_{h}\left(\phi_{h}, \omega_{h} ; \underline{\boldsymbol{\psi}}, \Pi_{1} z\right)-\mathcal{B}\left(\boldsymbol{\phi}_{h}, \omega_{h} ; \boldsymbol{\psi}, z\right)=a\left(\boldsymbol{\phi}_{h}, \underline{\boldsymbol{\psi}}-\boldsymbol{\psi}\right)+\left(\gamma_{h}, \nabla \underline{z}-\underline{\boldsymbol{\psi}}-\nabla z+\boldsymbol{\psi}\right)$

Using (5.23) and $(\boldsymbol{\gamma}, \boldsymbol{\psi}-\underline{\boldsymbol{\psi}})=a(\boldsymbol{\phi}, \boldsymbol{\psi}-\underline{\boldsymbol{\psi}})$, we obtain

$$
\begin{aligned}
& (g, z-\underline{z})+\left(\gamma_{h}, \nabla \underline{z}-\underline{\psi}-\nabla z+\boldsymbol{\psi}\right) \\
= & \left(\gamma_{h}-\gamma, \nabla \underline{z}-\underline{\psi}-\nabla z+\boldsymbol{\psi}\right)+(\boldsymbol{\gamma}, \boldsymbol{\psi}-\underline{\boldsymbol{\psi}}) \\
= & (\lambda \mu)^{-1} t^{2}\left(\boldsymbol{\gamma}-\boldsymbol{\gamma}_{h}, \mu \boldsymbol{s}-\hat{\boldsymbol{s}}\right)+a(\boldsymbol{\phi}, \boldsymbol{\psi}-\underline{\boldsymbol{\psi}}) .
\end{aligned}
$$

Combining the above four identities, we obtain

$$
\begin{aligned}
\left\|\phi-\phi_{h}\right\|_{0}^{2}+\left\|\omega-\omega_{h}\right\|_{0}^{2}= & a\left(\phi-\phi_{h}, \boldsymbol{\psi}-\underline{\boldsymbol{\psi}}\right)+(\lambda \mu)^{-1} t^{2}\left(\boldsymbol{\gamma}-\gamma_{h}, \mu \boldsymbol{s}-\hat{\boldsymbol{s}}\right) \\
& +\left(\lambda^{-1} t^{2} \gamma_{h}-\left(\nabla \omega_{h}-\phi_{h}\right), \boldsymbol{s}\right) .
\end{aligned}
$$

Using the local expression of $\gamma_{h}$, we get

$$
\begin{aligned}
\left(\lambda^{-1} t^{2} \boldsymbol{\gamma}_{h}-\left(\nabla \omega_{h}-\boldsymbol{\phi}_{h}\right), \boldsymbol{s}\right) & =\lambda^{-1} t^{2}(1-1 / \mu)\left(\gamma_{h}, \boldsymbol{s}\right)+\left(\nabla \mathcal{L}_{h} \boldsymbol{\phi}_{h}, \boldsymbol{s}\right) \\
& =\lambda^{-1} t^{2}(1-1 / \mu)\left(\boldsymbol{\gamma}_{h}, \boldsymbol{s}\right)-\left(\mathcal{L}_{h} \boldsymbol{\phi}_{h}, \operatorname{div} \boldsymbol{s}\right) .
\end{aligned}
$$

It follows from (4.30) that

$\left|\left(\lambda^{-1} t^{2} \gamma_{h}-\left(\nabla \omega_{h}-\phi_{h}\right), s\right)\right| \leq C\left(h\left\|\gamma-\gamma_{h}\right\|_{h}+h^{2}\|\gamma\|_{0}\right)\|s\|_{0}+C h^{2}\left\|\phi_{h}\right\|_{1}\|\operatorname{div} \boldsymbol{s}\|_{0}$.

Therefore, we obtain

$$
\begin{aligned}
\left\|\boldsymbol{\phi}-\boldsymbol{\phi}_{h}\right\|_{0}^{2}+\left\|\omega-\omega_{h}\right\|_{0}^{2} \leq & \lambda^{-1}\left\|\boldsymbol{\gamma}-\gamma_{h}\right\|_{h}\|\mu \boldsymbol{s}-\hat{\boldsymbol{s}}\|_{h}+C\left\|\boldsymbol{\phi}-\boldsymbol{\phi}_{h}\right\|_{1}\|\boldsymbol{\psi}-\underline{\boldsymbol{\psi}}\|_{1} \\
& +C\left(h\left\|\boldsymbol{\gamma}-\boldsymbol{\gamma}_{h}\right\|_{h}+h^{2}\|\boldsymbol{\gamma}\|_{0}\right)\|\boldsymbol{s}\|_{0}+C h^{2}\left\|\boldsymbol{\phi}_{h}\right\|_{1}\|\operatorname{div} \boldsymbol{s}\|_{0} .
\end{aligned}
$$

Invoking (5.17) $)_{1}$ for the MIN4 element, and (5.17) ${ }_{2}$ for the FMIN4 element, using Theorem 5.5 and the regularity estimate (5.15), we get (5.19) and (5.21).

We turn to the stabilized MITC4 element. Proceeding along the same way, we obtain an equation similar to (5.25):

$$
\begin{aligned}
\left\|\boldsymbol{\phi}-\boldsymbol{\phi}_{h}\right\|_{0}^{2}+\left\|\omega-\omega_{h}\right\|_{0}^{2}= & a\left(\boldsymbol{\phi}-\boldsymbol{\phi}_{h}, \boldsymbol{\psi}-\underline{\boldsymbol{\psi}}\right)+(\lambda \mu)^{-1} t^{2}\left(\boldsymbol{\gamma}-\boldsymbol{\gamma}_{h}, \mu \boldsymbol{s}-\hat{\boldsymbol{s}}\right) \\
& +(\boldsymbol{\gamma}, \underline{\boldsymbol{\psi}}-\Pi \underline{\boldsymbol{\psi}})+\left(\lambda^{-1} t^{2} \boldsymbol{\gamma}_{h}-\left(\nabla \omega_{h}-\boldsymbol{\phi}_{h}\right), \boldsymbol{s}\right) .
\end{aligned}
$$

Similar to (5.26), we have

$$
\begin{aligned}
\left(\lambda^{-1} t^{2} \gamma_{h}-\left(\nabla \omega_{h}-\phi_{h}\right), \boldsymbol{s}\right)= & \lambda^{-1} t^{2}(1-1 / \mu)\left(\boldsymbol{\gamma}_{h}, \boldsymbol{s}\right)+\left(\boldsymbol{\phi}_{h}-\Pi \boldsymbol{\phi}_{h}, \boldsymbol{s}\right) \\
= & \lambda^{-1} t^{2}(1-1 / \mu)\left(\boldsymbol{\gamma}_{h}, \boldsymbol{s}\right)+\left((\boldsymbol{I}-\Pi)\left(\boldsymbol{\phi}_{h}-\boldsymbol{\Pi}_{1} \boldsymbol{\phi}\right), \boldsymbol{s}\right) \\
& +\left(\boldsymbol{\Pi}_{1} \boldsymbol{\phi}-\Pi \boldsymbol{\Pi}_{1} \boldsymbol{\phi}, \boldsymbol{s}\right) .
\end{aligned}
$$


It follows from (5.12) and (4.35) that

$$
\begin{aligned}
\left|\left(\lambda^{-1} t^{2} \gamma_{h}-\left(\nabla \omega_{h}-\phi_{h}\right), s\right)\right| \leq C h\left(\left\|\boldsymbol{\phi}-\phi_{h}\right\|_{1}+\left\|\boldsymbol{\gamma}-\gamma_{h}\right\|_{h}\right)\|\boldsymbol{s}\|_{0} \\
+C h^{1+\alpha}\|\operatorname{rot} \boldsymbol{\phi}\|_{0}\|\boldsymbol{s}\|_{0}+C h^{2}\left(\|\boldsymbol{\phi}\|_{2}+\|\boldsymbol{\gamma}\|_{0}\right)\|\boldsymbol{s}\|_{\boldsymbol{H}(\mathrm{div})} .
\end{aligned}
$$

Using (4.35), we get

$$
|(\boldsymbol{\gamma}, \underline{\boldsymbol{\psi}}-\Pi \underline{\boldsymbol{\psi}})| \leq C\left(h^{2}\|\boldsymbol{\gamma}\|_{\boldsymbol{H}(\mathrm{div})}+h^{1+\alpha} t\|\boldsymbol{\gamma}\|_{0}\right)\left(\|\boldsymbol{\psi}\|_{2}+t^{-1}\|\operatorname{rot} \boldsymbol{\psi}\|_{0}\right) .
$$

Repeating the procedure for the MIN4 element, we come to (5.20).

\section{ERror estimate For a MOdified Q4BL ElEment}

The modified Q4BL element can be formulated as

Problem 6.1. Find $\left(\phi_{h}, \omega_{h}, \gamma_{h}\right) \in \boldsymbol{V}_{h}^{*} \times W_{h} \times M_{h}$ such that

$$
\mathcal{A}_{h}\left(\phi_{h}, \omega_{h}, \gamma_{h} ; \boldsymbol{\psi}, v, s\right)=\left(g, v+\mathcal{L}_{h} \boldsymbol{\psi}\right) \quad \forall(\boldsymbol{\psi}, v, \boldsymbol{s}) \in \boldsymbol{V}_{h}^{*} \times W_{h} \times \boldsymbol{M}_{h},
$$

where $\mathcal{A}_{h}\left(\phi_{h}, \omega_{h}, \gamma_{h} ; \boldsymbol{\psi}, v, \boldsymbol{s}\right)=\hat{\mathcal{A}}_{h}\left(\boldsymbol{\phi}_{h}, \omega_{h}+\mathcal{L}_{h} \boldsymbol{\phi}_{h}, \boldsymbol{\gamma}_{h} ; \boldsymbol{\psi}, v+\mathcal{L}_{h} \boldsymbol{\psi}, \boldsymbol{s}\right)$, and $\hat{\mathcal{A}}_{h}$ is defined for any $(\boldsymbol{\eta}, w, \boldsymbol{z})$ and $(\boldsymbol{\psi}, v, \boldsymbol{s}) \in \boldsymbol{V} \times W \times \boldsymbol{M}$ as

$$
\begin{aligned}
\hat{\mathcal{A}}_{h}(\boldsymbol{\eta}, w, \boldsymbol{z} ; \boldsymbol{\psi}, v, \boldsymbol{s}) & =\mathcal{A}(\boldsymbol{\eta}, w, z ; \boldsymbol{\psi}, v, \boldsymbol{s}) \\
& +\mu \lambda / t^{2}\left(\nabla w-\boldsymbol{\eta}-\lambda^{-1} t^{2} \boldsymbol{z}, \nabla v-\boldsymbol{\psi}-\lambda^{-1} t^{2} \boldsymbol{s}\right)
\end{aligned}
$$

with

$$
\mu_{\left.\right|_{K}}:=\mu_{K}=\rho t^{2} /\left(t^{2}+\alpha_{K} h_{K}^{2}\right) \quad \text { for some } \quad \rho \in(0,1) .
$$

Noting that for any $(\boldsymbol{\eta}, w, \boldsymbol{z}) \in \boldsymbol{V} \times W \times \boldsymbol{M}$,

$$
\hat{\mathcal{A}}_{h}(\boldsymbol{\eta}, w, \boldsymbol{z} ; \boldsymbol{\psi}, v, \boldsymbol{s})=\mu \mathcal{B}(\boldsymbol{\eta}, w ; \boldsymbol{\psi}, \boldsymbol{s})+(1-\mu) \mathcal{A}(\boldsymbol{\eta}, w, \boldsymbol{z} ; \boldsymbol{\psi}, v, \boldsymbol{s})
$$

for all $(\boldsymbol{\psi}, v, \boldsymbol{s}) \in \boldsymbol{V} \times W \times \boldsymbol{M}$.

Remark 6.2. The only difference between Problem 6.1 and the original formulation of Auricchio and Lovadina is the definition of $\mu$. We define a piecewise constant with a special form as in (6.2).

In view of Remark 5.1 and along the same line as [7, Proposition 3.1], we obtain the following weak coercivity inequality.

Lemma 6.3. Given $(\boldsymbol{\eta}, w, \boldsymbol{z}) \in \boldsymbol{V}_{h}^{*} \times W_{h} \times \boldsymbol{M}_{h}$, there exist $(\boldsymbol{\psi}, v, \boldsymbol{s}) \in \boldsymbol{V}_{h}^{*} \times W_{h} \times$ $\boldsymbol{M}_{h}$ such that $\|\boldsymbol{\psi}, v, \boldsymbol{s}\| \leq C \mid\|\boldsymbol{\eta}, w, \boldsymbol{z}\|$ and

$$
\mathcal{A}_{h}(\boldsymbol{\eta}, w, \boldsymbol{z} ; \boldsymbol{\psi}, v, \boldsymbol{s}) \geq C \mid\|\boldsymbol{\eta}, w, \boldsymbol{z}\|^{2} .
$$

With the above weak coercivity inequality, we derive the error bound as

Theorem 6.4. Let $(\boldsymbol{\phi}, \omega, \gamma)$ and $\left(\phi_{h}, \omega_{h}, \gamma_{h}\right)$ be solutions of Problems 2.1 and 6.1, respectively. If the $(1+\alpha)$-Section Condition holds, then

$$
\left\|\phi-\phi_{h}, \omega-\omega_{h}, \gamma-\gamma_{h}\right\| \leq C h\left(\|g\|_{-1}+t\|g\|_{0}\right)+C h^{2 \alpha}\|g\|_{-1} .
$$

Proof. For $\left(\phi_{h}-\Pi_{1} \phi, \omega_{h}-\Pi_{1} \omega, \gamma_{h}-\Pi_{0} \gamma\right) \in \boldsymbol{V}_{h}^{*} \times W_{h} \times M_{h}$, there exist $(\boldsymbol{\psi}, v, \boldsymbol{s}) \in$ $\boldsymbol{V}_{h}^{*} \times W_{h} \times \boldsymbol{M}_{h}$ such that $\|\boldsymbol{\psi}, v, \boldsymbol{s}\| \leq C\left\|\left|\boldsymbol{\phi}_{h}-\boldsymbol{\Pi}_{1} \boldsymbol{\phi}, \omega_{h}-\Pi_{1} \omega, \boldsymbol{\gamma}_{h}-\boldsymbol{\Pi}_{0} \gamma\right|\right\|$ and $\mathcal{A}_{h}\left(\phi_{h}-\Pi_{1} \phi, \omega_{h}-\Pi_{1} \omega, \gamma_{h}-\Pi_{0} \gamma ; \boldsymbol{\psi}, v, \boldsymbol{z}\right) \geq C\left\|\phi_{h}-\Pi_{1} \boldsymbol{\phi}, \omega_{h}-\Pi_{1} \omega, \gamma_{h}-\boldsymbol{\Pi}_{0} \boldsymbol{\gamma}\right\| \|^{2}$. 
On the other hand,

$$
\begin{aligned}
\mathcal{A}_{h}\left(\phi_{h}-\Pi_{1} \phi, \omega_{h}-\Pi_{1} \omega, \boldsymbol{\gamma}_{h}-\boldsymbol{\Pi}_{0} \boldsymbol{\gamma} ; \boldsymbol{\psi}, v, \boldsymbol{s}\right)= & \left(g, v+\mathcal{L}_{h} \boldsymbol{\psi}\right) \\
& -\mathcal{A}_{h}\left(\boldsymbol{\Pi}_{1} \boldsymbol{\phi}, \Pi_{1} \omega, \boldsymbol{\Pi}_{0} \boldsymbol{\gamma} ; \boldsymbol{\psi}, v, \boldsymbol{s}\right) .
\end{aligned}
$$

Note that $\hat{\mathcal{A}}_{h}\left(\boldsymbol{\phi}, \omega, \boldsymbol{\gamma} ; \boldsymbol{\psi}, v+\mathcal{L}_{h} \boldsymbol{\psi}, \boldsymbol{s}\right)=\left(g, v+\mathcal{L}_{h} \boldsymbol{\psi}\right)$ for any $(\boldsymbol{\psi}, v, \boldsymbol{s}) \in \boldsymbol{V}_{h}^{*} \times W_{h} \times$ $\boldsymbol{M}_{h}$. Hence,

$$
\begin{aligned}
& \mathcal{A}_{h}\left(\phi_{h}-\Pi_{1} \phi, \omega_{h}-\Pi_{1} \omega, \boldsymbol{\gamma}-\Pi_{0} \boldsymbol{\gamma} ; \boldsymbol{\psi}, v, s\right) \\
& =\hat{\mathcal{A}}_{h}\left(\boldsymbol{\phi}-\Pi_{1} \boldsymbol{\phi}, \omega-\Pi_{1} \omega-\mathcal{L}_{h} \Pi_{1} \boldsymbol{\phi}, \boldsymbol{\gamma}-\Pi_{0} \boldsymbol{\gamma} ; \boldsymbol{\psi}, v+\mathcal{L}_{h} \boldsymbol{\psi}, \boldsymbol{s}\right) .
\end{aligned}
$$

By virtue of (6.3), the above identity can be expanded into

$$
\begin{aligned}
& \mathcal{A}_{h}\left(\phi_{h}-\boldsymbol{\Pi}_{1} \boldsymbol{\phi}, \omega_{h}-\Pi_{1} \omega, \boldsymbol{\gamma}-\boldsymbol{\Pi}_{0} \boldsymbol{\gamma} ; \boldsymbol{\psi}, v, \boldsymbol{s}\right)=a\left(\boldsymbol{\phi}-\boldsymbol{\Pi}_{1} \boldsymbol{\phi}, \boldsymbol{\psi}\right) \\
& +\left(\mu \boldsymbol{\gamma}-\widehat{\boldsymbol{\gamma}}, \nabla\left(v+\mathcal{L}_{h} \boldsymbol{\psi}\right)-\boldsymbol{\psi}\right)+(1-\mu)\left(\boldsymbol{\gamma}-\boldsymbol{\Pi}_{0} \boldsymbol{\gamma}, \nabla\left(v+\mathcal{L}_{h} \boldsymbol{\psi}\right)-\boldsymbol{\psi}\right) \\
& +\mu^{-1}(1-\mu) \lambda^{-1} t^{2}(\mu \boldsymbol{\gamma}-\widehat{\boldsymbol{\gamma}}, \boldsymbol{s})-\lambda^{-1} t^{2}(1-\mu)\left(\boldsymbol{\gamma}-\boldsymbol{\Pi}_{0} \boldsymbol{\gamma}, \boldsymbol{s}\right) \\
& =: I_{1}+\cdots+I_{5} .
\end{aligned}
$$

We bound $I_{1}, \cdots, I_{5}$ separately. $I_{1}$ can be bounded as

$$
\left|I_{1}\right| \leq C h\|\phi\|_{2}\|\boldsymbol{\psi}\|_{1} .
$$

As in (5.16), we estimate $I_{2}$ as

$$
\left|I_{2}\right| \leq C\|\mu \boldsymbol{\gamma}-\widehat{\gamma}\|_{h}|\boldsymbol{\psi}, v|_{h} \leq C\left(\|\boldsymbol{\gamma}-\widehat{\gamma}\|_{h}+h\|\boldsymbol{\gamma}\|_{0}\right)|\boldsymbol{\psi}, v|_{h} .
$$

$I_{3}$ can be bounded as

$$
\left|I_{3}\right| \leq\left\|\gamma-\Pi_{0} \gamma\right\|_{h}|\boldsymbol{\psi}, v|_{h} .
$$

Similar to $I_{2}$, we bound $I_{4}$ as

$$
\left|I_{4}\right| \leq C\left(\|\gamma-\widehat{\gamma}\|_{h}+h\|\gamma\|_{0}\right)\|s\|_{h} .
$$

Clearly, $I_{5}=0$. To sum up, we obtain

$$
\left\|\phi_{h}-\Pi_{1} \phi, \omega_{h}-\Pi_{1} \omega, \gamma_{h}-\Pi_{0} \gamma\right\| \leq C h\left(\|g\|_{-1}+t\|g\|_{0}\right)+C h^{2 \alpha}\|g\|_{-1} .
$$

A combination of (6.5) and Lemma 5.2 leads to the conclusion.

In the following, we turn to the $\mathrm{L}^{2}$ estimate for this modified Q4BL element.

Theorem 6.5. Let $(\phi, \omega, \gamma)$ and $\left(\phi_{h}, \omega_{h}, \gamma_{h}\right)$ be solutions of Problems 2.1 and 6.1, respectively. If the $(1+\alpha)$-Section Condition holds, then

$$
\left\|\boldsymbol{\phi}-\boldsymbol{\phi}_{h}\right\|_{0}+\left\|\omega-\omega_{h}\right\|_{0} \leq C\left(h^{2}+h^{1+2 \alpha}\right)\left(\|g\|_{-1}+t\|g\|_{0}\right)+C h^{4 \alpha}\|g\|_{-1} .
$$

Proof. The solution of Problem 5.6] satisfies

$$
\hat{\mathcal{A}}_{h}(\boldsymbol{m}, n, \boldsymbol{p} ; \boldsymbol{\psi}, z, \boldsymbol{s})=\left(\boldsymbol{\phi}-\boldsymbol{\phi}_{h}, \boldsymbol{m}\right)+\left(\omega-\omega_{h}, n\right) \quad \forall(\boldsymbol{m}, n, \boldsymbol{p}) \in \boldsymbol{V} \times W \times \boldsymbol{M} .
$$

Substituting $\boldsymbol{m}=\boldsymbol{\phi}-\boldsymbol{\phi}_{h}, n=\omega-\omega_{h}$, and $\boldsymbol{p}=\gamma-\gamma_{h}$ into (6.7), we obtain

$$
\begin{aligned}
\left\|\boldsymbol{\phi}-\phi_{h}\right\|_{0}^{2}+ & \left\|\omega-\omega_{h}\right\|_{0}^{2}=\hat{\mathcal{A}}_{h}\left(\boldsymbol{\phi}-\boldsymbol{\phi}_{h}, \omega-\omega_{h}, \boldsymbol{\gamma}-\gamma_{h} ; \boldsymbol{\psi}, z, \boldsymbol{s}\right) \\
= & \hat{\mathcal{A}}_{h}\left(\boldsymbol{\phi}-\boldsymbol{\phi}_{h}, \omega-\omega_{h}-\mathcal{L}_{h} \boldsymbol{\phi}_{h}, \boldsymbol{\gamma}-\gamma_{h} ; \boldsymbol{\psi}-\underline{\boldsymbol{\psi}}, z-\underline{z}, \boldsymbol{s}-\underline{\boldsymbol{s}}\right) \\
& +\hat{\mathcal{A}}_{h}\left(\boldsymbol{\phi}-\boldsymbol{\phi}_{h}, \omega-\omega_{h}-\mathcal{L}_{h} \boldsymbol{\phi}_{h}, \boldsymbol{\gamma}-\gamma_{h} ; \underline{\boldsymbol{\psi}}, \underline{z}, \underline{\boldsymbol{s}}\right) \\
& +\hat{\mathcal{A}}_{h}\left(\mathbf{0}, \mathcal{L}_{h} \boldsymbol{\phi}_{h}, \mathbf{0} ; \boldsymbol{\psi}, z, \boldsymbol{s}\right)
\end{aligned}
$$

with $\underline{\boldsymbol{\psi}}:=\Pi_{1} \boldsymbol{\psi}, \underline{z}:=\Pi_{1} z+\mathcal{L}_{h} \underline{\boldsymbol{\psi}}$, and $\underline{\boldsymbol{s}}:=\Pi_{0} s$. Note that

$$
\hat{\mathcal{A}}_{h}(\boldsymbol{\phi}, \omega, \boldsymbol{\gamma} ; \underline{\boldsymbol{\psi}}, \underline{z}, \underline{\boldsymbol{s}})=(g, \underline{z})=\hat{\mathcal{A}}_{h}\left(\phi_{h}, \omega_{h}+\mathcal{L}_{h} \phi_{h}, \boldsymbol{\gamma}_{h} ; \underline{\boldsymbol{\psi}}, \underline{z}, \underline{\boldsymbol{s}}\right)
$$


and $\hat{\mathcal{A}}_{h}\left(\mathbf{0}, \mathcal{L}_{h} \boldsymbol{\phi}_{h}, \mathbf{0} ; \boldsymbol{\psi}, z, \boldsymbol{s}\right)=\left(\nabla \mathcal{L}_{h} \boldsymbol{\phi}_{h}, \boldsymbol{s}\right)$. Therefore,

$$
\begin{aligned}
\left\|\phi-\phi_{h}\right\|_{0}^{2}+\left\|\omega-\omega_{h}\right\|_{0}^{2}= & \hat{\mathcal{A}}_{h}\left(\boldsymbol{\phi}-\phi_{h}, \omega-\omega_{h}-\mathcal{L}_{h} \phi_{h}, \boldsymbol{\gamma}-\gamma_{h} ; \boldsymbol{\psi}-\underline{\boldsymbol{\psi}}, z-\underline{z}, \boldsymbol{s}-\underline{\boldsymbol{s}}\right) \\
& +\left(\nabla \mathcal{L}_{h} \phi_{h}, \boldsymbol{s}\right) .
\end{aligned}
$$

We need to consider the cross term

$$
\mu \lambda t^{-2}\left(\nabla \omega-\boldsymbol{\phi}-\nabla\left(\omega_{h}+\mathcal{L}_{h} \phi_{h}\right)+\phi_{h}, \nabla z-\boldsymbol{\psi}-\nabla \underline{z}+\underline{\psi}\right) .
$$

This term can be rewritten as $\left(\nabla \omega-\boldsymbol{\phi}-\nabla\left(\omega_{h}+\mathcal{L}_{h} \boldsymbol{\phi}_{h}\right)+\boldsymbol{\phi}_{h}, \mu \boldsymbol{s}-\hat{\boldsymbol{s}}\right)$, which is in turn bounded by

$$
C\left\|\lambda \mu t^{-2}\left(\nabla \omega-\phi-\nabla\left(\omega_{h}+\mathcal{L}_{h} \phi_{h}\right)+\phi_{h}\right)\right\|_{h}\|\mu \boldsymbol{s}-\hat{\boldsymbol{s}}\|_{h} .
$$

It follows from (4.30) that

$$
\left|\left(\nabla \mathcal{L}_{h} \phi_{h}, s\right)\right|=\left|-\left(\mathcal{L}_{h} \phi_{h}, \operatorname{div} s\right)\right| \leq C h^{2}\left\|\phi_{h}\right\|_{1}\|\operatorname{div} \boldsymbol{s}\|_{0} .
$$

The remaining terms of $\hat{\mathcal{A}}_{h}$ can be easily bounded, and thus we obtain

$$
\begin{aligned}
\left\|\boldsymbol{\phi}-\boldsymbol{\phi}_{h}\right\|_{0}^{2}+\left\|\omega-\omega_{h}\right\|_{0}^{2} \leq & C\left\|\boldsymbol{\phi}-\boldsymbol{\phi}_{h}, \omega-\omega_{h}, \boldsymbol{\gamma}-\boldsymbol{\gamma}_{h}\right\|+C h^{2}\left\|\boldsymbol{\phi}_{h}\right\|_{1}\|\operatorname{div} \boldsymbol{s}\|_{0} \\
+ & C\left\|\lambda \mu t^{-2}\left(\nabla \omega-\boldsymbol{\phi}-\nabla\left(\omega_{h}+\mathcal{L}_{h} \boldsymbol{\phi}_{h}\right)+\phi_{h}\right)\right\|_{h} \\
& \times\left(\|\boldsymbol{\psi}-\underline{\boldsymbol{\psi}}\|_{1}+\|\boldsymbol{s}-\underline{\boldsymbol{s}}\|_{h}+\|\mu \boldsymbol{s}-\hat{\boldsymbol{s}}\|_{h}\right) .
\end{aligned}
$$

Using Remark 5.1, we get

$$
\begin{aligned}
\| \lambda \mu t^{-2}\left(\nabla\left(\omega_{h}+\mathcal{L}_{h} \phi_{h}\right)\right. & \left.-\phi_{h}-\nabla\left(\Pi_{1} \omega+\mathcal{L}_{h} \Pi_{1} \phi\right)+\Pi_{1} \phi\right) \|_{h} \\
& \leq C\left|\phi_{h}-\Pi_{1} \phi, \omega_{h}-\Pi_{1} \omega\right|_{h}+C\left\|\phi_{h}-\Pi_{1} \phi\right\|_{1} .
\end{aligned}
$$

Using $\lambda \mu t^{-2}\left(\nabla \omega-\phi-\nabla\left(\Pi_{1} \omega+\mathcal{L}_{h} \Pi_{1} \phi\right)+\Pi_{1} \phi\right)=\mu \gamma-\widehat{\gamma}$ and (5.16), we obtain

$$
\begin{gathered}
\left\|\lambda \mu t^{-2}\left(\nabla \omega-\phi-\nabla\left(\Pi_{1} \omega+\mathcal{L}_{h} \Pi_{1} \phi\right)+\Pi_{1} \phi\right)\right\|_{h}=\|\mu \boldsymbol{\gamma}-\widehat{\gamma}\|_{h} \\
\leq C\left(\|\gamma-\widehat{\gamma}\|_{h}+h\|\gamma\|_{0}\right) .
\end{gathered}
$$

By the triangle inequality, using (6.5) and the interpolation estimate (5.9) $)_{1}$, we get

$$
\left\|\mu t^{-2}\left(\nabla \omega-\phi-\nabla\left(\omega_{h}+\mathcal{L}_{h} \phi_{h}\right)+\phi_{h}\right)\right\|_{h} \leq C h\left(\|g\|_{-1}+t\|g\|_{0}\right)+C h^{2 \alpha}\|g\|_{-1} .
$$

Substituting the above inequality into (6.8), using Theorem 6.4 and the interpolation estimate (5.4), (5.17) 1 , we obtain the desired $\mathrm{L}^{2}$ error estimate (6.6).

Remark 6.6. The modified Q4BL element could attain the optimal $H^{1}$ and $L^{2}$ error if we employ the full kinematically linked interpolation operator $\widetilde{\mathcal{L}}_{h}$ in (6.1).

\section{Conclusion}

Theorems 5.5, 5.7, 6.4 and 6.5 indicate that the FMIN4 and the stabilized MITC4 elements are more robust than the MIN4 and the modified Q4BL elements from the viewpoint of the mesh distortion. The $(1+\alpha)$-Section Condition, instead of the commonly used Bi-Section Condition, distinguishes the convergence behavior of the MIN4 and the modified Q4BL from the stabilized MITC4 and the FMIN4. The robustness of these elements with respect to the mesh distortion can be expressed as

$$
\text { FMIN4 > STABILIZED MITC4 > MIN4, MODIFIED Q4BL. }
$$

The degradation of the accuracy of these four elements over the general quadrilateral mesh is actually not widely known. It is also difficult to detect from numerous numerical reports presented in [27, 35, 39] since they all employed bisection as a mesh refinement strategy, and the resulting mesh as addressed in [1, 31] is always 
the asymptotically regular parallelogram mesh even if the initial mesh is the one as shown in the right figure of Figure 1. For these meshes, our theoretic results show that there is no accuracy loss for all four elements.

\section{ACKNOWLEDGMENTS}

This work was initiated when the first author visited the Faculty of Mathematics at the Ruhr-University Bochum on a grant by the Alexander von Humboldt Foundation. He thanks the Alexander von Humboldt Foundation for their support. The work of the first author was partially supported by the National Natural Science Foundation of China 10201033, 10571172 and also supported by the National Basic Research Program under the grant 2005CB321704.

We are grateful to the anonymous referees for improving the presentation of this paper.

\section{REFERENCES}

1. D. N. Arnold, D. Boffi and R. S. Falk, Approximation by quadrilateral finite elements, Math. Comp. 71 (2002), 909-922. MR1898739 (2003c:65112)

2. D. N. Arnold, D. Boffi and R. S. Falk, Quadrilateral $\boldsymbol{H}$ (DIV) finite elements, SIAM J. Numer. Anal., 42 (2005), 2429-2451. MR2139400 (2006d:65129)

3. D. N. Arnold, D. Boffi, R. S. Falk and L. Gastaldi, Finite element approximation on quadrilateral meshes, Communications in Numerical Methods in Engineering 17 (2001), 805-812. MR.1872639(2002i:65121)

4. D. N. Arnold and F. Brezzi, Some new elements for the Reissner-Mindlin plate model, Boundary Value Problems for Partial Differential Equations and Applications (J. L. Lions and C. Baiocchi, eds.), Masson, 1993, pp. 287-292. MR1260452 (94k:73066)

5. D. N. Arnold and R. S. Falk, A uniformly accurate finite element method for the ReissnerMindlin plate, SIAM J. Numer. Anal. 26 (1989), 1276-1290. MR.1025088 (91c:65068)

6. D. N. Arnold and R. S. Falk, Asymptotic analysis of the boundary layer for the ReissnerMindlin plate model, SIAM J. Math. Anal. 27 (1996), 486-514. MR1377485 (97i:73064)

7. F. Auricchio and C. Lovadina, Partial selective reduced integration schemes and kinematically linked interpolations for plate bending problems, Math. Mod. Meth. Appl. Sci. 9 (1999), 693722. MR.1697391 (2001c:74080)

8. R. Ayad, G. Dhatt and J. L. Batoz, A new hybrid-mixed variational approach for ReissnerMindlin plates: The MiSP model, Internat. J. Numer. Methods Engrg. 42 (1998), 1149-1179. MR.1633100 (99d:73085)

9. R. Ayad and A. Rigolot, An improved four-node hybrid-mixed element based upon Mindlin's plate theory, Internat. J. Numer. Methods Engrg. 55 (2002), 705-731.

10. K.-J. Bathe, Finite Element Procedure, Prentice-Hall, 1996. MR0851177 (87j:65144)

11. K.-J. Bathe and E. Dvorkin, A four-node plate bending element based on Mindlin/Reissner plate theory and a mixed interpolation, Internat. J. Numer. Methods Engrg. 21 (1985), 367383.

12. S. C. Brenner and L. R. Scott, The Mathematical Theory of Finite Element Methods, SpringerVerlag, 1994. MR:1278258 (95f:65001)

13. F. Brezzi and M. Fortin, Numerical approximation of Mindlin-Reissner plates, Math. Comp. 47 (1986), 151-158. MR0842127 (87g:73057)

14. F. Brezzi and M. Fortin, Mixed and Hybrid Finite Element Methods, Springer-Verlag, New York, 1991. MR.1115205(92d:65187)

15. D. Chapelle and R. Stenberg, An optimal low-order locking-free finite element method for Reissner-Mindlin plates, Math. Mod. Meth. Appl. Sci. 8 (1998), 407-430. MR1624871 (99d:73088)

16. P. G. Ciarlet, The Finite Element Method for Elliptic Problems, North-Holland, Amsterdam, 1978. MR0520174 (58 \#25001)

17. R. Duran, E. Hernández, L. Hervella-Nieto, E. Liberman and R. Rodríguez, Error estimates for lower-order isoparametric quadrilateral finite elements for plates, SIAM J. Numer. Anal. 41 (2003), 1751-1772. MR2035005 (2004m:65192) 
18. I. Fried and S. K. Yang, Triangular, nine-degrees-of-freedoms, $\mathrm{C}^{0}$ plate bending element of quadratic accuracy, Quart. Appl. Math. 31 (1973), 303-312.

19. V. Girault and P.-A. Raviart, An analysis of the finite element method for Navier-Stokes equations, Numer. Math. 33 (1979), 235-271. MR0553589 (81a:65100)

20. T. J. R. Hughes, The Finite Element Methods: Linear Static and Dynamic Finite Element Analysis, Prentice-Hall, Englewood, Cliffs, NJ 1987. MR1008473 (90i:65001)

21. F. Kikuchi and K. Ishii, An improved 4-node quadrilateral plate bending element of the Reissner-Mindlin type, Comput. Mech. 23 (1999), 240-249.

22. A. M. Lakhany, I. Marek and J. R. Whiteman, Superconvergence results on mildly structured triangulation, Comput. Methods Appl. Mech. Engrg. 189 (2000), 1-75. MR1779678 (2001f:74041)

23. C. Lovadina, A new class of mixed finite element for Reissner-Mindlin plate, SIAM J. Numer. Anal. 33 (1996), 2457-2467. MR.1427474 (98e:73136)

24. M. Lyly, On the connection between some linear triangular Reissner-Mindlin plate bending elements, Numer. Math. 85 (2000), 77-107. MR751364 (2001b:65127)

25. M. Lyly and R. Stenberg, The stabilized MITC plate bending elements, Proceedings of the Fourth World Conference on Computational Mechanics. Buenos Aires, June-July, 1998. MR 1839065

26. M. Lyly and R. Stenberg, Stabilized finite element methods for Reissner-Mindlin plates, preprint, 2001.

27. M. Lyly, R. Stenberg and T. Vihinen, A stable bilinear element for Reissner-Mindlin plates, Comput. Methods Appl. Mech. Engrg. 110 (1993), 343-357. MR1256325 (94k:73072)

28. P. B. Ming and Z.-C. Shi, Nonconforming rotated $\mathrm{Q}_{1}$ finite element for Reissner-Mindlin plate, Math. Mod. Meth. Appl. Sci. 11 (2001), 1311-1342. MR.1859825 (2002h:65191)

29. P. B. Ming and Z.-C. Shi, Optimal L ${ }^{2}$ error bounds for MITC3 type element, Numer. Math. 91 (2002), 77-91. MR1896088 (2003e:65225)

30. P. B. Ming and Z.-C. Shi, Optimal L ${ }^{2}$ error estimate for stabilized MITC4 element, preprint, 2001.

31. P. B. Ming and Z.-C. Shi, Quadrilateral mesh revisited, Comput. Methods Appl. Mech. Engrg. 191 (2002), 5671-5682. MR 1941167 (2003h:65169)

32. P. B. Ming and Z.-C. Shi, Two nonconforming quadrilateral elements for the Reissner-Mindlin plate, Math. Mod. Meth. Appl. Sci., 15 (2005), 1503-1517. MR.2168943

33. J. Pitkäranta, Analysis of some low-order finite element schemes for Mindlin-Reissner and Kirchhoff plates, Numer. Math. 53 (1988), 237-254. MR0946378 (89f:65126)

34. Z.-C. Shi, A convergence condition for the quadrilateral Wilson element, Numer. Math. 44 (1984), 349-361. MR0757491 (86d:65151)

35. A. Tessler and T. J. R. Hughes, An improved treatment of transverse shear in the Mindlin type four-node quadrilateral element, Comput. Methods Appl. Mech. Engrg. 39 (1983), 311-335.

36. J. M. Thomas, Sur l'analyse numérique des méthods d'éléments finis hybrides et mixtes, Thése d'Etat, Université Pierre et Marie Curie, Paris, 1977.

37. Z. Xu, A simple and efficient triangular finite element for plate bending, Acta Mech. Sin. 2 (1986), 185-192.

38. Z. Xu, O. C. Zienkiewicz and L. F. Zhang, Linked interpolation for Reissner-Mindlin plate elements, III, an alternative quadrilateral element, Internat. J. Numer. Methods Engrg. 37 (1994), 1437-1443.

39. O. C. Zienkiewicz, Z. Xu, L. F. Zeng, A. Samuelsson and N. E. Wiberg, Linked interpolation for Reissner-Mindlin plate elements, I: A simple quadrilateral, Internat. J. Numer. Methods. Engrg. 36 (1993), 3043-3056.

LSEC, Institute of Computational Mathematics and Scientific/Engineering Computing, AMSS, Chinese Academy of Sciences, No. 55, Zhong-Guan-Cun East Road, Beijing, 100080, People's Republic of China

E-mail address: mpb@lsec.cc.ac.cn

LSEC, Institute of Computational Mathematics and Scientific/Engineering Computing, Amss, Chinese Academy of Sciences, No. 55, Zhong-Guan-Cun East Road, Beijing, 100080, People's Republic of ChinA

E-mail address: shi@lsec.cc.ac.cn 